\title{
Genome-wide analysis of the lignin toolbox for morus and the roles of lignin related genes in response to zinc stress
}

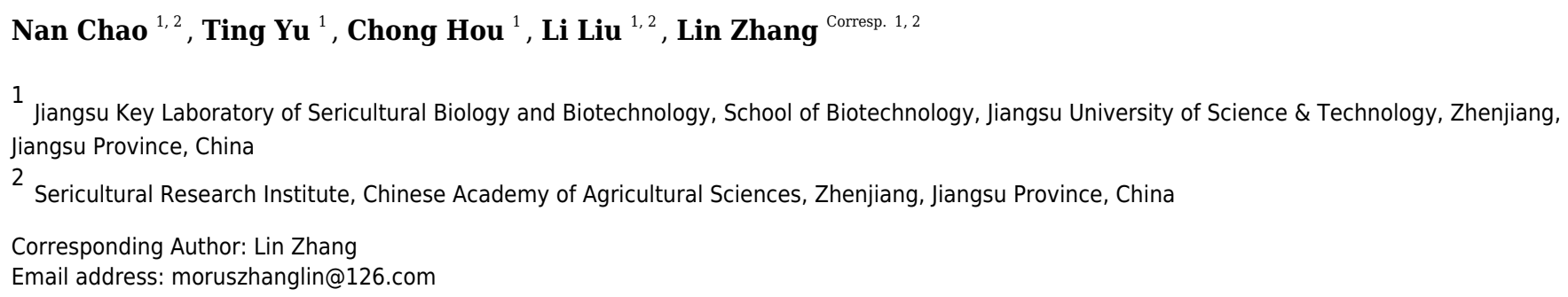

Mulberry (Morus, Moraceae) is an important economic plant with nutritional, medicinal, and ecological values. Lignin in mulberry can affect the quality of forage and the saccharification efficiency of mulberry twigs. The availability of the Morus notabilis genome makes it possible to perform a systematic analysis of the genes encoding the 11 protein families specific to the lignin branch of the phenylpropanoid pathway, providing the core genes for the lignin toolbox in mulberry. We performed genome-wide screening, which was combined with de novo transcriptome data for Morus notabilis and Morus alba variety Fengchi, to identify putative members of the lignin gene families followed by phylogenetic and expression profile analyses. We focused on bona fide clade genes and their response to zinc stress were further distinguished based on expression profiles using RNA-seq and RT-qPCR. We finally identified 31 bona fide genes in Morus notabilis and 25 bona fide genes in Fengchi. The putative function of these bona fide genes was proposed, and a lignin toolbox that comprised 19 genes in mulberry was provided, which will be convenient for researchers to explore and modify the monolignol biosynthesis pathway in mulberry. We also observed changes in the expression of some of these lignin biosynthetic genes in response to stress caused by excess zinc in Fengchi and proposed that the enhanced lignin biosynthesis in lignified organs and inhibition of lignin biosynthesis in leaf is an important response to zinc stress in mulberry. 
1 Genome-wide analysis of the lignin toolbox for morus and the roles of lignin

3 Nan Chao ${ }^{1,2}$, Ting Yu ${ }^{1}$, Chong Hou ${ }^{1}$, Li Liu ${ }^{1,2}$, Lin Zhang ${ }^{1,2 \dagger}$,

4 1. Jiangsu Key Laboratory of Sericultural Biology and Biotechnology, School of Biotechnology,

5 Jiangsu University of Science and Technology, Zhenjiang, Jiangsu 212018, China

6 2. Key Laboratory of Silkworm and Mulberry Genetic Improvement, Ministry of Agriculture and

7 Rural Affairs, Sericultural Research Institute, Chinese Academy of Agricultural Sciences, 8 Zhenjiang, Jiangsu 212018, China

$\dagger$ To whom correspondence should be addressed: Tel (Fax): +86 51185616638

E-mail: Zhanglinsri@126.com, Jiangsu University of Science and Technology.

The English in this document has been checked by at least two professional editors, both native speakers of English. Letpub has provided the Linguistical service for this MS. 
16 Abstract: Mulberry (Morus, Moraceae) is an important economic plant with nutritional, 17 medicinal, and ecological values. Lignin in mulberry can affect the quality of forage and the

saccharification efficiency of mulberry twigs. The availability of the Morus notabilis genome makes it possible to perform a systematic analysis of the genes encoding the 11 protein families specific to the lignin branch of the phenylpropanoid pathway, providing the core genes for the lignin toolbox in mulberry. We performed genome-wide screening, which was combined with de novo transcriptome data for Morus notabilis and Morus alba variety Fengchi, to identify putative members of the lignin gene families followed by phylogenetic and expression profile analyses. We focused on bona fide clade genes and their response to zinc stress were further distinguished based on expression profiles using RNA-seq and RT-qPCR. We finally identified 31 bona fide genes in Morus notabilis and 25 bona fide genes in Fengchi. The putative function of these bona fide genes was proposed, and a lignin toolbox that comprised 19 genes in mulberry was provided, which will be convenient for researchers to explore and modify the monolignol biosynthesis pathway in mulberry. We also observed changes in the expression of some of these lignin biosynthetic genes in response to stress caused by excess zinc in Fengchi and proposed that the enhanced lignin biosynthesis in lignified organs and inhibition of lignin biosynthesis in leaf is an important response to zinc stress in mulberry.

Key words: gene family, genome-wide, lignin, mulberry, zinc stress 


\section{Introduction}

Lignin is an important component of plant cell walls and has important functions in plant growth and stress resistance (Chun, et al. 2019). In turn, owing to its recalcitrant nature and complexity, lignin limits the efficient conversion of lignocellulosic biomass to ethanol (Ragauskas, et al. 2014). The modification of trees with less lignin or with more-degradable lignin along with normal growth, which can improve the quality of forage and saccharification efficiency, has become a hot topic (Dixon, et al. 2014; Umezawa 2018).

The lignin biosynthesis pathway has been deciphered and revised since its discovery decades ago (Whetten and Sederoff 1995). As of now, a total of 11 enzymes have been identified to play a role in monolignol biosynthesis (Zhao 2016). The monolignol biosynthesis pathway generally refers to the branch of phenylpropanoid pathway starting with the deamination of phenylalanine and leading to the production of hydroxycinnamyl alcohols. The general phenylpropanoid pathway contains phenylalanine ammonia-lyase (PAL), cinnamate 4-hydroxylase $(\mathrm{C} 4 \mathrm{H})$ and 4coumarate: CoA ligase (4CL) and provides hydroxycinnamoyl-CoA esters as precursors for a wide range of end products, including lignin, flavonoids, anthocyanins and condensed tannins. In the monolignol-specific biosynthesis pathway, hydroxycinnamoyl-CoA esters undergo successive hydroxylation and O-methylation of their aromatic rings, as well as redox reactions, to produce the monolignols (Zhao 2016). Coumaroyl shikimate 3'-hydroxylase (C3'H) and ferulate 5hydroxylase $(\mathrm{F} 5 \mathrm{H})$ are responsible for the hydroxylation process. Shikimate $O$-hydroxycinnamoyl -transferase (HCT), caffeoyl CoA 3-O-methyltransferase (CCoAOMT) and caffeate/5- 
55 hydroxyferulate $O$-methyltransferase (COMT) are involved in the O-methylation process.

56 Caffeoyl shikimate esterase (CSE) was recently discovered to convert caffeoyl shikimate into

57 caffeate and consists of a bypass with 4CL (Saleme, et al. 2017; Vanholme, et al. 2013). Redox

58 reactions are catalyzed successively by cinnamoyl CoA reductase (CCR) and cinnamyl alcohol

59 dehydrogenase (CAD) to achieve the conversion of the side-chain carboxyl to an alcohol group.

60 CCR and CAD constitute the primary pathway for monolignol biosynthesis (Zhao 2016).

61 Mulberry (Morus, Moraceae) is an important economic plant in Asia with considerable

62 nutritional and medicinal values (Yuan and Zhao 2017). Moraceae is one of the closest relatives

63 of Rosaceae and mulberry diverged from Cannabis sativa (Cannabaceae) 63.5 Mya, from

64 apple/strawberry (Rosaceae) 88.2 Mya and from Medicago truncatula (Fabales) 101.6 Mya (He,

65 et al. 2013; Jiao, et al. 2020). Many studies have shown the great potential of this plant in the

66 energy, food and pharmaceutical industries. Mulberry has long been cultivated for sericulture,

67 which shaped the world's history through the Silk-Road. Furthermore, a large number of by-

68 products of branches twigs have been produced from the large-scale cultivation of mulberry trees

69 in traditional sericulture, and mulberry has been gradually considered a potentially new energy

70 plant providing biomass for the production of biofuels (Łochyńska 2015; Tang, et al. 2012).

71 Studies of lignin biosynthesis have been widely reported for energy plants and forage plants, such

72 as poplar, Medicago sativa L. and Eucalyptus grandis (Carocha, et al. 2015; Hamberger, et al.

73 2007; Lee, et al. 2011; Shi, et al. 2010). Recently, Wang et al. characterized four Ma4CL genes 
74 from M. atropurpurea $c v$. Jialing No. 40. and revealed the functional divergence of Ma4CL

75 (Wang, et al. 2016).

76 The availability of the Morus notabilis genome and an increasing number of transcriptomic data

77 for mulberry allows comprehensive genome-wide analyses of lignin biosynthesis genes in this

78 species (Li, et al. 2014). In addition, a recent study has been reported to reveal the chromosome-

79 level genome of Morus alba (Jiao, et al. 2020). Genome-wide screening, combined with de novo

80 transcriptome data, was performed in this study on Morus notabilis and Morus alba variety

81 Fengchi to obtain the genes putatively included in the 11 monolignol gene families. M. alba is one

82 of the most widely cultivated mulberry in China. M. alba variety Fengchi is a new variety created

83 by Sericultural Research Institute, Chinese Academy of Agricultural Sciences, expected to spread

84 and grow in extreme environment conditions and used as heavy metal hyperaccumulators and

85 forage. A phylogenetic tree and expression profile were used to further identify the bona fide genes

86 involved in lignin biosynthesis, and finally, we provided a lignin toolbox consisting of 19 genes in

87 Morus notabilis and 17 genes in Morus alba variety Fengchi, which will be convenient for

88 researchers to explore and modify the monolignol biosynthesis pathway in this genus. We also

89 assessed the potential roles of lignin biosynthetic genes in response to stress caused by the excess

90 of zinc in Fengchi and proposed that the promotion of lignification in lignifying organs, associated

91 with the inhibition of lignin deposition in leaves, is an important response to zinc stress in

92 mulberry.

\section{Materials and Methods}


94

95

96

97

98

99

100

101

102

103

104

105

106

107

108

109

110

111

112

113

114

\section{Plant Materials}

The materials used in this study were obtained from the National Germplasm Resource Nursery of the Institute of Sericulture, Chinese Academy of Agricultural Sciences. Annual seedlings, Morus alba L. variety Fengchi, were transplanted into plastic flowerpots, and the potted plants were irrigated with $400 \mathrm{ml} / \mathrm{kg}$ of Murashige and Skoog (MS) medium to provide nutrients (Susheelamma, et al. 1996). Zinc sulfate powder was applied near the roots of the mulberry trees as excess zinc stress treatment $(450 \mathrm{mg} / \mathrm{kg})$. Changes of proline and superoxide dismutase (SOD) concentration were determined on the 15th day (Figure S1). The root, stem and leaf tissues were quickly frozen in liquid nitrogen and stored at $-80^{\circ} \mathrm{C}$. This experiment was performed using three biological replicates. These collected samples were used for both RNA-seq and RT-qPCR (quantitative real-time PCR) analysis.

\section{Genome-wide screening of candidate genes for the lignin toolbox in mulberry}

Bona fide genes involved in lignin biosynthesis with functional characterization from different plants were collected as a query sequence for an HMMer search using MorusDB online ((https://morus.swu.edu.cn /morusdb) (Li, et al. 2014). The sequence identity $(>45 \%)$, e-value $(<\mathrm{e}-$ $100)$ and full score $(>400)$ were used to screen for candidate gene family members. For some gene family members such as CSE and CCoAOMT, the thresholds were flexible to obtain as many as possible candidate genes. A local blastp search was also performed to identify the Selaginella moellendorffii SmF5H and Fengchi homologs (Christiam, et al. 2009). All of the query sequences used and candidate genes obtained are available in Table S1.

\section{De novo transcriptome assembly of Morus alba variety Fengchi}


115 Transcriptome de novo assembly was carried out with the short reads assembling program Trinity

116 (Grabherr, et al. 2011). Unigenes were aligned by BLASTx $(\mathrm{e}<0.00001)$ to protein databases in

117 nr, Swiss-Prot, KEGG and COG/KOG. The best alignment results were chosen to determine the

118 sequence direction of unigenes. When a unigene could not be aligned to any of these protein

119 databases, the protein coding sequence and sequence direction were confirmed using ESTscan

120 (Iseli, et al. 1999). The data set is available with accession number PRJNA660559 in National

121 Center for Biotechnology Information (NCBI)

122 Sequence alignment and phylogenetic analysis

123 Putative protein sequences of different plant species were used for alignment and phylogenetic

124 analysis. Sequences used for phylogenetic analysis were screened from various sources based on

125 the platform PLAZA 3.0 (http://bioinformatics.psb.ugent.be/plaza/). Sequences from the

gymnosperm Picea sitchensis and the fern Selaginella moellendorffii were obtained using BlastP

in the NCBI database. Bona fide lignin related genes in different plants were obtained based on

published studies (Carocha, et al. 2015; Raes, et al. 2003). Alignment was performed using

DNAman 8.0 (Lynnon BioSoft) with default parameters. Phylogenetic trees were constructed

using Mega 7.0 with the maximum-likelihood method (Kumar, et al. 2016). The phylogenetic tree

was assessed by bootstrapping using 1000 bootstrap replicates and marked above nodes only if

greater than 50 . The JTT substitution model and $\mathrm{G}+\mathrm{I}$ rates among sites model were selected as

parameters for building the tree. The putative protein sequences used are listed in Table S2.

\section{Expression profile analysis}

135 The gene expression based on the large-scale transcriptome data was calculated and normalized to 
136

137

138

139

140

141

142

143

144

145

146

147

148

149

150

151

152

153

154

155

156

RPKM (reads per kb per million reads). Transcriptome data of different tissues and organs (root, bark, leaves, winter bud, male-flower) in Morus notabilis was obtained from Mrousdb

(https://morus.swu.edu.cn /morusdb) (Li, et al. 2014). RNA-seq data for Fengchi different organs

(root, stem and leaf) was aligned to de-novo transcriptome assembly of Morus alba variety

Fengchi using bowtie2 and RPKM values for unigenes were calculated using deptools v2.0 based

on the bam files (Langdon 2015; Ramirez, et al. 2014). RT-qPCR (quantitative real-time PCR)

was also performed to validate gene expressions of 23 bona fide clade genes in different organs

and the change of their expression levels after zinc treatment using ABI StepOnePlus ${ }^{\mathrm{TM}}$ Real-Time

PCR System (USA). Genes that showed preferential expression in lignifying tissue or organs (bark,

root and stem) were considered as candidate lignin-related genes. Primers based on the coding sequences of these genes were designed using Primer-Blast. The primers are available in Table

S3 and the melt curve of each gene is provided in Figure S2. Actin was used as reference gene according to previous studies (Shukla, et al. 2019). Tbtools was used to visualize the expression profile (Chen, et al. 2020), and Graphpad Prism8.0 was used to visualize the RT-qPCR results. SPSS19.0 was used to perform T-test and ANOVA, $\mathrm{p}<0.05$ was marked as significant. Three biological replicates were considered for transcriptome data and two biological replicates with three technical replicates respectively were performed for RT-qPCR.

\section{Results}

\section{Genome-wide screening of monolignol biosynthesis pathway-related genes}

Morusdb (https://morus.swu.edu.cn/morusdb), which provides the genome and transcriptome information for Morus notabilis, was used to perform genome-wide screening of candidate genes 
157 involved in monolignol biosynthesis. Finally, we obtained 56 candidate genes based on the

158 HMMer search and blastp results (Table S1). In addition, we identified their homologs in Fengchi,

159 a Morus alba variety bred by our institute, based on our de novo transcriptome data. Most (49/56)

160 of the corresponding homologs were identified in Fengchi using candidate genes in Morus

161 notabilis as a reference sequence.

162 Phenylalanine Ammonia-Lyase (PAL)

163 PAL (EC: 4.3.1.5) catalyzes the deamination of phenylalanine to produce cinnamic acid and is the 164 initial step in the general phenylpropanoid pathway. We constructed a phylogenetic tree (Figure 1A) using both (Morus notabilis $\mathrm{Mn}$ ) MnPAL and (Fengchi Fc) FcPAL and bona fide PAL data reported in other species. Seven MnPALs were identified and clustered as bona fide PALs. However, only three homologs, FcPAL3, FcPAL6 and FCPAL7, were found in Fengchi based on de novo transcriptome data. MnPAL7 and FcPAL7 were quite divergent compared with other

PALs in angiosperms and are closer to PALs from gymnosperms, which is similar to EgrPAL2 in

Eucalyptus grandis (Carocha, et al. 2015). Both MnPAL7 and FCPAL7 showed a low expression

level in various tissues and organs and exhibited no obvious preference in the lignified tissues and

organs (Figure 1B). MnPAL1, 2, 4, and 5 are phylogenetically close to AtPAL1 and AtPAL2,

which have been reported to be mainly involved in anthocyanin production (Cochrane, et al. 2004;

Huang, et al. 2010). MnPAL1 and 5 were preferentially expressed in lignifying organs and tissues

175 (root and bark), which differed from the expression patterns of MnPAL2 and 4 (Figure 1B).

MnPAL1, 2, 4, and 5 (L484_024371, L484_024373, L484_024372, L484_024369) have high sequence identity (Aligned protein sequence identity>99\%) and are located close to each other in 
178 the genome, forming a gene cluster. Although MnPAL1, 2, 4, and 5 showed high expression levels 179 in the studied organs and tissues in Morus notabilis, we could not find or distinguish homologs of 180 MnPAL1, 2, 4, and 5 in Fengchi. Mn PAL3, 6 and FcPAL3, 6 are phylogenetically close to AtPAL4, 5 and PtrPAL4, 5, which are reported to express more specifically in xylem tissues (Raes, et al. 2003). MnPAL3 and $F c P A L 3$ also showed an expression preference in the root, stem or bark, with a high overall expression level, while MnPAL6 and FcPAL6 showed low overall expression levels in all of the examined organs and tissues (Figure 1B). RT-qPCR results also validated the expression preference of $F c P A L 3$ in stems (Figure S3). Based on the above facts, MnPAL1, 3,5 and $F C P A L 3$ are the PAL genes most likely to be involved in lignification.

\section{4-Coumaric acid coenzyme A ligase (4CL)}

4CL (EC: 6.2.1.12) belongs to the ANL (AMP-producing adenylating superfamily of enzymes) superfamily and catalyzes the formation of CoA thiol esters of 4-coumarate and other 4hydroxycinnamates, which are important input metabolites, especially for lignin biosynthesis and flavonoid biosynthesis (Ehlting, et al. 1999). The bona fide 4CL clade in angiosperm comprises three classes. Clade I contains 4CLs, which are mainly involved in lignin biosynthesis, including

At4CL1, 3, 4, Pto4CL1, 3, 4, 5, Mn4CL1, 2, 4 and Fc4CL1, 2, 4 (Figure 1C). The expression profiles of $M n 4 C L 1,2,4$ and $F C 4 C L 1,2,4$ also indicated preferential expression in the root, stem or bark (Figure 1D and Figure S3). Mn4CL3 and Fc4CL3 were clustered in Clade II together with At4CL3, Pto4CL2 and Os4CL2, which have been reported to be associated with flavonoid and soluble phenolic biosynthesis (Gui, et al. 2011; Li, et al. 2015; Rao, et al. 2015). Mn4CL3 showed 
199 its possible function in flavonoid biosynthesis. The third clade only contained Os4CLs 200 (Os4CL1/3/4/5), which are thought to be distinct from the lignin-associated clade I 4CLs found in 201 dicots. We also found that Mn4CL5, 6 and Fc4CL5, 6 were in a separate cluster and were

202

203

204 205 206 207 208 209

210

phylogenetically close to (Plagiochasma appendiculatum) Pa4CL, the liverwort Plagiochasma appendiculatum. Mn4CL5 showed a similar expression pattern to Mn4CL3 and a high expression in male flowers and bark. Mn4CL6 had an expression specific to male flowers. These facts indicate that Mn4CL5, 6 may also be involved in flavonoid and soluble phenolic biosynthesis, given the high flavonoid content in mulberry. Mn4CL5, 6 and Fc4CL5, 6 are divergent from 4CLs in angiosperms and still need to be further studied to identify their roles in mulberry. Therefore, Mn4CL1, 2, 3, 4 and FC4CL1, 2, 3, 4 are the 4CL genes most likely to be involved in lignification.

\section{Hydroxylation steps in the general phenylpropanoid pathway}

C4H (EC: 1.14.13.11) and C3'H (EC: 1.14.14.1) catalyze the hydroxylation steps. C4H and C3'H belong to the CYP73 and CYP98 families, respectively which are members of the cytochrome P450 monooxygenase superfamily. $\mathrm{C} 4 \mathrm{H}$ is generally encoded by small gene family, except in Arabidopsis, which has only one AtC4H. Studies in Populus have shown a C4H-C3'H complex that more efficiently catalyzes hydroxylation steps (Chen, et al. 2011). Here, we identified three candidate $\mathrm{C} 4 \mathrm{Hs}$ in mulberry. MnC4H1, 2 and $\mathrm{FcC} 4 \mathrm{H} 1,2$ clustered with AtC4H and PoptrC4H1, 2 as Clade I, which is responsible for lignin biosynthesis (Figure 2A). $M n C 4 H 1,2$ showed a high expression in all organs and tissues. $F c C 4 H 1,2$ was preferentially expressed in lignified organs (Figure 2C, Figure S3). MnC4H3 and FcC4H3 were grouped with PoptrC4H3 and are distinct from MnC4H1, 2 and FcC4H1, 2. Similar to PoptrC4H3, MnC4H3 and FcC4H3 had a very low 
220

221

222

223

224

225

226

227

228

229

230

231

232

233

234

235

236

237

238

239

240

expression level in all of the studied organs. Therefore, $M n C 4 H 1,2$ and $F c C 4 H 1,2$ are the $C 4 H$ genes most likely to be involved in lignification.

Although C3'H was shown to catalyze the conversion of $p$-coumaric acid into caffeic acid in vitro, further studies demonstrated that its activity in vitro is the conversion of $p$-coumaroyl shikimate to caffeoyl shikimate (Abdulrazzak, et al. 2006; Franke, et al. 2002, Schoch et al. 2001). Based on our phylogenetic analysis, only MnC3'H1 and $\mathrm{FcC} 3$ 'H1 clustered with $\mathrm{StC} 3$ 'Hs as bona fide clade II (Figure 2B). It is interesting to note that $\mathrm{MnC}$ ' $\mathrm{H} 1$ and $\mathrm{FcC} 3 \mathrm{H} 1$ are phylogenetically closer to C3'Hs in monocots, other than C3'Hs such as AtC3'H and PoptrC3'H in dicots (Figure 2B). Other candidates, including $\mathrm{MnC} 3$ ' $\mathrm{H} 2-5$ or $\mathrm{FcC} 3$ ' $\mathrm{H} 2-5$, is in a separate cluster without any C3'H orthologs in other plants. It seems that $\mathrm{C} 3$ ' $\mathrm{H}$ in mulberry is similar to that in A. thaliana, which also has only one C3'H (Raes et al., 2003). MnC3'H1showed a high expression in all of the studied organs, and $F c C 3$ 'H1 was preferentially expressed in the stem, according to both transcriptome data and RT-qPCR, which likely involves lignification (Figure 2C and Figure S3).

Therefore, $M n C 3$ ' $H 1$ and $F c C^{\prime} 3 H 1$ are the $C 3 H$ genes most likely to be involved in lignification.

\section{Hydroxycinnamoyl CoA: shikimate hydroxycinnamoyl transferase (HCT) and caffeoyl}

\section{shikimate esterase (CSE)}

HCT (EC: 2.3.1.133) combined with C3'H (p-coumarate 3-hydroxylase) catalyzes two steps to change the carbon flux from $\mathrm{H}$ to $\mathrm{G}$ and $\mathrm{S}$ lignin units. HCT belongs to the BAHD acyltransferase family and is able to utilize a variety of non-native substrates (Chiang, et al. 2018;

D’Auria 2006). P-coumaroyl-CoA and caffeoyl-CoA are preferential substrates for HCTs, and HCTs catalyze the acylation of CoA esters with shikimate, producing shikimate esters containing 
241

242 caffeoyl shikimate is also catalyzed by HCT. Hydroxy-cinnamoyl CoA: quinate

243 hydroxycinnamoyl transferase (HQT) is another acyl transferase that uses quinic acid instead of

244 shikimic acid as the acceptor compound and is involved in chlorogenic acid biosynthesis, not lignin

coumaric acid or caffeic acid. The reverse reaction for the formation of caffeoyl-CoA from biosynthesis (Niggeweg, et al. 2004). We constructed a phylogenetic tree using both HCTs and HQTs to distinguish bona fide HCT clades (Figure 3A). Six candidate MnHCTs were grouped as bona fide HCTs with HCTs in angiosperms. MnHCT2, 3 (L484_000457, L484_018078) and MnHCT5, 6 (L484_017530, L484_017529) had high sequence similarity (aligned protein sequence identity $>95 \%$ ). We could not distinguish FCHCT2, 3 and FCHCT5, 6 based only on transcripts; therefore, we named $F c H C T 2$ and 5 based on their similar expression pattern to MnHCT2 and MnHCT5. Among all MnHCTs, MnHCT1, 2 and FCHCT1, 2 were preferentially expressed in lignified organs and tissues (stems, roots and bark) and are likely involved in monolignol biosynthesis (Figure 3C. Figure S3). Other MnHCTs and FcHCTs showed relatively low expression in all organs and tissues. MnHCT5, 6 and FcHCT5 are phylogenetically divergent from other MnHCTs and MnHCTs and showed preferential expression in the leaf (FCHCT5) and in the winter bud and leaf (MnHCT5), which indicates their possible different roles as opposed to lignification in mulberry. Therefore, $M n H C T 1,2$ and $F c H C T 1,2$ are the $H C T$ genes most likely to be involved in lignification.

AtCSE was first characterized as lysoPL2, a member of the monoacylglycerol lipase (MAGL) gene family in Arabidopsis (Kim et al. 2016; Gao et al. 2010). AtCSE was first reported as caffeoyl shikimate esterase by Vanholme et al. (2013) in Arabidopsis because of its ability to convert 
262

263

264

265

266

267

268

269

270

271

272

273

274

275

276

277

278

279

280

281

282

caffeoyl shikimate into caffeate. Further analysis of an A. thaliana cse-2 (caffeoyl shikimate esterase 2) knockout mutant that presented a reduced lignin content enriched in $\mathrm{H}$ units and depleted in S units indicated the involvement of CSE (EC: 3.1.1.) in lignin biosynthesis (Vanholme, et al. 2013). CSE competes with HCT for the substrate caffeoyl shikimate. MnCSE1 and FcCSE1 are phylogenetically close to AtCSE, PoptrCSE1,2 and MtCSE, which have been reported to be involved in lignin biosynthesis (Figure 3B) (Ha, et al. 2016; Saleme, et al. 2017). In addition, $F c C S E 1$ showed preferential expression in lignified organs and tissues (Figure 3C, Figure S3). MnCSE2 and FcCSE2 showed close relationship with AtGAML1 which was reported to harbor MAG lipase activities and lysophosphatidylcholine (LPC) and/or lysophosphatidy lethanolamine (LPE) hydrolase activities. MnCSE3, without a homolog in Fengchi, was far from the bona fide CSEs and cluster with AtMAGL9 and 12. MnCSE3 had an expression preference in winter buds and male flowers. In general, $M n C S E 1$ and $F c C S E 1$ are lignin-related CSEs, but MnCSE2 and FcCSE2 are monoacylglycerol lipase.

\section{The methylation steps}

COMT (EC: 2.1.1.68) and CCoAOMT (EC: 2.1.1.104) are both involved in the methylation steps of the monolignol pathway (Zhong, et al. 1998). CCoAOMT catalyzes the methylation of caffeoyl CoA to produce feruloyl CoA and is reported to be responsible for $\mathrm{G}$ and S-type lignin. Only one CCoAOMT in mulberry, MnCCoAOMT1 or FcCCoAOMT1, clusters in the bona fide clade with AtCCoAOMT and PtoCCoAOMT1 and 2 (Figure 4A). Both MnCCoAOMTl and FcCCoAOMT1 showed a high expression level in the lignified organs (Figure 4C, Figure S3). FcCCoAOMT1 had the highest expression level in the stems, about 50-fold higher than that in the leaves (Figure 4C). 
$283 \mathrm{MnCCoAOMT1}$ had high expression in the root, bark and male flowers, with the highest expression

284 in the root (two-fold higher than the expression in the bark or male flower, five-fold higher than

285 the expression in the leaf). Two other candidates, MnCCoAOMT2, 3 and FcCCoAOMT2, 3 belong

286 to the CCOAOMT-like clade and had a low expression level in all organs and tissues. Therefore,

287 MnCCOAOMT1 and FcCCoAOMT1 are the CCoAOMT1 genes most likely to be involved in

288 lignification.

289 In angiosperms, COMT (EC: 2.1.1.68) is involved in the synthesis S precursors and is now

considered to be primarily involved in the synthesis of S units through the preferential methylation

of 5-hydroxyconiferyl aldehyde into sinapaldehyde based on functional analysis in several species

(Davin, et al. 2008) In mulberry, only one COMT, MnCOMT4 or FcCOMT4 was identified as a

bona fide COMT together with AtCOMT and PoptrCOMT1,2, based on our phylogenetic analysis

(Figure 4B). MnCOMT4 and FcCOMT4 showed obvious expression preference in the lignified

organs and tissues (Figure 4C, Figure S3) and should be responsible for lignin biosynthesis in

mulberry. Other candidate MnCOMTs and FcCOMTs were in a separate cluster and

phylogenetically far from the bona fide clade. MnCOMT2, 5 showed a relatively high expression

in male flowers compared with that in the leaf, winter bud and bark. MnCOMT1 and 6 showed a

very low expression in all of the detected organs, and the RPKM of MnCOMT6 based on the

published transcriptome data is not available. FcCOMT1, 2, 3, 5, and 6 had similar expression

patterns, with overall low expression in all organs and a relatively high expression in roots

compared with the stem and leaf. These COMT-like genes need more evidence and functional

analysis for elucidating their roles in mulberry. Therefore, MnCOMT4 and FcCOMT4 are the 
304

305

306

307

308

309

310

311

312

313

314

315

316

317

318

319

320

321

322

323

324

COMT genes most likely to be involved in lignification.

\section{Hydroxylation step specific for $\mathbf{S}$ lignin production}

F5H (EC:1.14.13) belongs to the CYP84 family and is similar to $\mathrm{C} 3 \mathrm{H}$ and $\mathrm{C} 4 \mathrm{H}$ as a member of the cytochrome P450 monooxygenases. F5H (EC: 1.14.13), also called CAld5H because of its substrate preference for coniferaldehyde/coniferyl alcohol (Humphreys, et al. 1999), catalyzes the hydroxylation step specific for the production of sinapyl alcohol and, ultimately, S lignin. The discovery of $\mathrm{SmF} 5 \mathrm{H}$ in the lycophyte Selaginella moellendorffii revealed a novel P450 (CYP788A1) (Weng, et al. 2008). SmF5H shares only 37\% amino acid sequence identity with its angiosperm counterparts and can also use $p$-coumaraldehyde and $p$-coumaryl alcohol as substrates to efficiently produce caffeoyl aldehyde and caffeoyl alcohol. Therefore, SmF5Hs can divert Gsubstituted intermediates toward S lignin synthesis through related but distinct pathways compared with angiosperms (Weng and Chapple 2010). In addition to the genome-wide screening using F5Hs from angiosperms, we carried out blastp using $\mathrm{SmF} 5 \mathrm{H}$ as a query to find more F5H-like sequences MnF5H1, 2 and FcF5H1, 2 were identified as candidate F5H based on a Hmmer search using F5Hs from angiosperms. MnF5H1 and FcF5H1 clustered with AtF5H and PoptrF5H1, 2 and belong to the bona fide clade in angiosperms (Figure 5A). MnF5H1 and FcF5H1 showed obvious expression preference in lignified organs and tissues and are likely to be involved in lignin biosynthesis (Figure 5B, Figure S3). In contrast, $M n F 5 H 2$ and $F c F 5 H 2$ were far from the bona fide clade and had very low expressions in all organs and tissues. Other candidate F5Hs named MnF5H3(Sm), MnF5H4(Sm) or FcF5H3(Sm), FcF5H4(Sm) were identified, sharing about 45\% protein sequence identity with SmF5H. MnF5H3(Sm), MnF5H4(Sm) and FcF5H3(Sm), 
325

326

327

328

329

330

331

332

333

334

335

336

337

338

339

340

341

342

343

344

345

$\mathrm{FcF} 5 \mathrm{H} 4(\mathrm{Sm})$ showed relatively high expression in the root, and $\mathrm{MnF5H3}(\mathrm{Sm})$ also had a high

overall expression in the bark, male flowers and leaf. These SmF5H-like proteins in mulberry may

be involved in the response to zinc stress since $F c F 5 H 3(\mathrm{Sm})$ and $F c F 5 H 4(\mathrm{Sm})$ both showed a decreased expression in the leaf after zinc treatment (Figure S4). Therefore, $M n F 5 H 1$ and FcF5H1 are the $F 5 H$ genes most likely to be involved in lignification.

\section{The last two reductive steps}

CCR (EC: 1.2.1.44) is the first committed enzyme for a specific branch of monolignol biosynthesis and converts various cinnamoyl-CoA esters ( $p$-coumaroyl-CoA, caffeoyl-CoA, feruloyl-CoA and sinapoyl-CoA) to produce their corresponding hydroxycinnamaldehydes, which are further reduced into different monolignols by another reductase called cinnamyl-alcohol dehydrogenase (CAD EC: 1.1.1.195). CCR and CAD are involved in the primary pathway of monolignol biosynthesis. A recent study showed that PoptrCAD1 and PoptrCCR2 can form a complex to regulate monolignol biosynthesis in Populus (Yan, et al. 2019).

Six candidate CCRs from the mulberry genome were screened; phylogenetic analysis showed that MnCCR1, 2 and FcCCR1, 2 belonged to the bona fide clade with AtCCR1,2, MtCCR1,2 and

PtoCCR1,7 (Figure 6A). Further motif-aware analysis based on our previously reported workflow further validated that MnCCR1,2 and FcCCR1,2 belonged to bona fide CCRs and other candidate CCRs should be CCR-like (Figure S5) (Chao, et al. 2019). Similar to AtCCR1, 2 and PtoCCR1, 7, different bona fide CCRs in mulberry also had different expression patterns. MnCCR1 and FCCCR 1 had a high overall expression, with the highest expression level in the bark or stem, while $M n C C R 2$ and $F c C C R 2$ had quite low expression in all organs and tissues (Figure 6C, Figure S3). 
346 Therefore, $M n C C R l$ and $F c C C R l$ are likely to play a predominant role in monolignol biosynthesis.

347 As both phylogenetic analysis and motif-aware analysis showed, other MnCCRs and FcCCRs

348 belong to the CCR-like cluster with unknown functions.

349 CAD is the last enzyme in monolignol biosynthesis and uses various phenylpropenyl aldehyde derivatives as substrates to ensure the diversity of lignin. We obtained six candidate MnCADs, and the corresponding homologs in Fengchi were found except MnCAD4. FcCAD4 was different from all six candidate MnCADs, with high (73.88\%) protein sequence identity to AtCAD1 which was reported to have very low catalytic activity in vitro and play roles in lignification of elongating stems (Eudes, et al. 2006; Kim, et al. 2004). MnCAD1, 2, 3, 4, 5 and FcCAD1, 2, 3, 5 belong to bona fide clades (Figure 5B). MnCAD3, 4 and FcCAD3 clustered with AtCAD4, 5, PtoCAD1, and BdCAD5, which have been reported to be involved in lignin biosynthesis. Although the expression data for MnCAD3, 4 were not available in Morusdb, FcCAD3 showed expression specific to lignified organs based on our transcriptome data and RT-qPCR (Figure 5C, Figure S3).

In addition to the above bona fide CADs, there is another kind of bona fide CAD with the present

PtrSAD in Populus (Li, et al. 2001). This PtrSAD has been reported to prefer sinapaldehyde as a substrate,however, our previous study on PtoCAD2 showed no obvious substrate preference (Chao, et al. 2014). MnCAD1,2 and FcCAD1, 2 are phylogenetically close to the so-called PtrSAD and PtoCAD2 and cluster as another bona fide clade. MnCAD1 showed no obvious expression preference while $F c C A D 1$ exhibit a preference for lignified organs based on RT-qPCR results

(Figure 5C, Figure S3). MnCAD2 and $F c C A D 2$ showed an expression preference for winter-bud or leaf (Figure 5C, Figure S3). 
367

368

369

370

371

372

373

374

375

376

377

378

379

380

381

382

383

384

385

386

387

\section{Summary of the lignin toolbox for mulberry and the response to zinc ion stress}

Genes considered the lignin toolbox in mulberry were annotated in TableS4. Hierarchical clustering depicted a similar expression pattern for bona fide lignin biosynthetic genes we described above, which differed from that of genes identified as 'like' genes" (candidate genes excluded from bona fide clade) (Figure S6 A). It was obvious that the bona fide genes had a higher overall expression in the studied organs than the 'like' genes. 21 of total 31 bona fide genes in mulberry can be classified as two main clusters based on their expression patterns. Cluster I (indicated as a blue star $\mathrm{r}$ ) includes genes with obvious expression preferences in lignified organs such as stem and bark, and cluster II (indicated as a red star) includes genes with a high expression but no obvious preference in lignified organs and tissues. RT-qPCR also validated the expression preference in lignified organs for the lignin-related genes comprise the lignin toolbox for mulberry (Figure S6B, Figure S3 and Table S4).

Mulberry has been reported as heavy metal hyperaccumulators. Our results showed that lignin-related genes play important roles in responding to zinc stress. All we detected bona fide clade genes (22/23) including 16 core genes in lignin toolbox in Fengchi show significant expression change in at least one organ (Figure 7A). Only FCCCOAOMT1 showed no significant change in any detected organs after zinc excess treatment (Figure 7A, Figure S3, Table S4). Monolignol biosynthesis pathway in Fengchi showed overall up-regulation in root and stem but down-regulation in leaf (Figure 7B). Most of these bona fide clade genes (13/22) were downregulated in leaves. Five genes showed up-regulation in both leaf and lignified organs (Figure 7C).

It is likely that the promotion of lignin biosynthesis in lignified organs in mulberry is an important 
388

389

390

391

392

393

394

395

396

397

398

399

400

401

402

403

404

405

406

407

408

way to respond to zinc stress.

\section{Discussion}

\section{Lignification toolbox in mulberry}

Genes involving in secondary metabolism in mulberry were reported to have faster evolutionary rate (Jiao, et al. 2020). Lignin biosynthesis is important pathway in land plants. Genome-wide screening of candidate genes involving in monolignol biosynthesis was performed here in mulberry. In total, 31 bona fide clade genes were obtained in Morus notabilis based on phylogenetic analysis, and 25 bona fide homologs were found in Fengchi, similar to Populus (25) and E. grandis (38) (Table S4) (Carocha, et al. 2015; Shi, et al. 2010). The loss of MnHCT and MnPAL homologs in Fengchi resulted in the above-mentioned change in the total numbers. The bona fide genes described above had a similar expression pattern and a higher overall expression in the studied organs compared with genes identified as 'like' genes. Combined with the expression profile in different organs of Morus notabilis and Fengchi, a total of 19 genes were identified as bona fide lignification-related genes, which is similar to that in E. grandis (17). These 19 genes were preferentially expressed in the lignified organs and tissues and probably represent the core lignification toolbox in mulberry (Figure S6B, Figure S3, Table S4).

\section{The lignin biosynthesis pathway plays an important role in the response to stress caused by}

\section{excess zinc in mulberry}

Zinc is a trace element that is necessary for a healthy immune system and is important for people to maintain their fitness level. Studies have shown that dietary zinc can act as sleep modulator and is necessary for brain development and function (Cherasse and Urade 2017; 
409 Hambidge 2000). Mulberry is a woody plant with resistance to zinc ions, and both leaves and fruits 410 of mulberry are known as sites rich in zinc (Jiang, et al. 2017; Srivastava, et al. 2006). Black 411 mulberry (Morus nigra) juice has high amounts of zinc and iron, which could help to improve the 412 micronutrient status of pregnant women and children (Khalid, et al. 2011) A deficiency or excess 413 zinc leads to oxidative stress. Moreover, the Zn-deficiency leads to abnormal development of 414 leaves in mulberry (Kumar Tewari, et al. 2008).

415 Mulberry is able to uptake the heavy metal and was reported to immigrate $254532.8 \mathrm{mg} \mathrm{Zn}$

416

417

418

419

420

421

422

424

425

426

427

428

every square meter plough layer soil (Jiang, et al. 2017). The contents of zinc in different mulberry organs (leaf, root, bark and stem) are greatly different (Jiang, et al. 2017). After excess zinc treatment, 23 core genes involved in lignin biosynthesis except CCoAOMT1 showed obvious expression changes in different organs (Figure 7). Monolignol biosynthesis pathway in Fengchi showed overall up-regulation in root and stem but down-regulation in leaf (Figure 7B). Relatively high expression of lignin related genes (total 24 genes) was also reported in response to zinc exposure in roots of Thlaspi caerulescens, one of the natural zinc hyperaccumulator species (van de Mortel, et al. 2006). Lignin has been reported to act as a metal-absorbing matrix in response to metal stress (Bhardwaj, et al. 2014). It is likely that the promotion of lignin biosynthesis in lignified organs association with inhibition of lignin biosynthesis in leaf in mulberry is an important response to zinc stress. A similar situation was reported for Lens Culinaris and Phaseolus Mungo subjected to lead stress (Haider and Azmat 2012).

\section{Supplementary Data}


429 Figure S1 Pro concentration and SOD concentration in mulberry leaves, roots and stems at 43015 days after treatment

431 Figure S2 The melt curve of each gene for RT-qPCR experiments

432 Figure S3 RT-qPCR results for 23 bona fide clade genes in Fengchi

433 Figure S4 RT-qPCR for FcF5H3(Sm) and FcF5H4(Sm) in Fengchi

434 Figure S5 Alignment and motif analysis of CCRs. The motifs were marked in red box.

435 Figure S6 Expression profile of all candidate genes in mulberry.

436 Table S1 Genome-wide screening of monolignon biosynthesis pathway-related genes

437 Table S2 Sequence used for lignin-related gene analysis in mulberry

438 Table S3 Primers used for RT-qPCR

439 Table S4 Putative functional classification of 56 candidate lignification-related genes

440 Table S5 Raw data for RT-qPCR

441 Table S6 Basic annotation of all unigenes

442 Acknowledgments

443 This work was jointly supported by the Crop Germplasm Resources Protection Project of the 444 Ministry of Agriculture and Rural Affairs of the People's Republic of China (19190172), National 445 Infrastructure for Crop Germplasm Resources (NICGR-2019-43), China Agriculture Research 446 System of MOF and MARA and Jiangsu Province Postgraduate Research and Practice Innovation 447 Project (KYCX20_3166).

\section{Conflict of Interests}

449 The authors declare that the research was conducted in the absence of any commercial or financial 450 relationships that could be construed as a potential conflict of interest. 


\section{Author Contribution}

452 Lin Zhang, Li Liu and Nan Chao guided the work and provided advices; Nan Chao and Ting Yu

453 performed the experiments; Nan Chao analyzed the data, organized the figures and wrote the

454 manuscript. Chong Hou performed proof-reading.

455 Availability of data and material

456 The raw sequencing data has been deposited in NCBI: https://www.ncbi.nlm.nih.

457 gov/sra/PRJNA660559. This Transcriptome Shotgun Assembly project has been deposited at

458

459

460

461

462

463

464

465

466

467

468

469

470

471

472

473

474

475

476

477

478

479

480

481

482

DDBJ/ENA/GenBank under the accession GJFU00000000. The version described in this paper is

the first version, GJFU01000000.

\section{References}

Abdulrazzak N, Pollet B, Ehlting J, Larsen K, Asnaghi C, Ronseau S, Proux C, Erhardt M, Seltzer V, Renou JP, Ullmann P, Pauly M, Lapierre C, Werck-Reichhart D (2006) A coumaroyl-ester-3-hydroxylase insertion mutant reveals the existence of nonredundant meta-hydroxylation pathways and essential roles for phenolic precursors in cell expansion and plant growth. Plant Physiol 140:30-48

Bhardwaj R, Handa N, Sharma R, Kaur H, Kohli S, Kumar V, Kaur P (2014) Lignins and Abiotic Stress: An Overview. In: Physiological Mechanisms and Adaptation Strategies in Plants Under Changing Environment. pp 267-296

Carocha V, Soler M, Hefer C, Cassan-Wang H, Fevereiro P, Myburg AA, Paiva JA, Grima-Pettenati J (2015) Genomewide analysis of the lignin toolbox of Eucalyptus grandis. New Phytol 206:1297-1313

Chao N, Jiang WT, Wang XC, Jiang XN, Gai Y (2019) Novel motif is capable of determining CCR and CCR-like proteins based on the divergence of CCRs in plants. Tree Physiol 39:2019-2026

Chao N, Liu SX, Liu BM, Li N, Jiang XN, Gai Y (2014) Molecular cloning and functional analysis of nine cinnamyl alcohol dehydrogenase family members in Populus tomentosa. Planta 240:1097-1112

Chen C, Chen H, Zhang Y, Thomas HR, Frank MH, He Y, Xia R (2020) TBtools - an integrative toolkit developed for interactive analyses of big biological data. Mol Plant

Chen HC, Li Q, Shuford CM, Liu J, Muddiman DC, Sederoff RR, Chiang VL (2011) Membrane protein complexes catalyze both 4- and 3-hydroxylation of cinnamic acid derivatives in monolignol biosynthesis. Proc Natl Acad Sci U S A 108:21253-21258

Chiang YC, Levsh O, Lam CK, Weng JK, Wang Y (2018) Structural and dynamic basis of substrate permissiveness in hydroxycinnamoyltransferase (HCT). PLoS Comput Biol 14:e1006511

Christiam, Camacho, George, Coulouris, Vahram, Avagyan, Ning, Ma, Jason, Papadopoulos (2009) BLAST+: architecture and applications. BMC Bioinformatics

Chun HJ, Baek D, Cho HM, Lee SH, Jin BJ, Yun DJ, Hong YS, Kim MC (2019) Lignin biosynthesis genes play critical roles

PeerJ reviewing PDF | (2020:12:56482:4:0:NEW 5 Jul 2021) 
483

484

485

486

487

488

489

490

491

492

493

494

495

496

497

498

499

500

501

502

503

504

505

506

507

508

509

510

511

512

513

514

515

516

517

518

519

520

521

522

523

in the adaptation of Arabidopsis plants to high-salt stress. Plant Signal Behav 14:1625697

Cochrane FC, Davin LB, Lewis NG (2004) The Arabidopsis phenylalanine ammonia lyase gene family: kinetic characterization of the four PAL isoforms. Phytochemistry 65:1557-1564

Davin LB, Jourdes M, Patten AM, Kim KW, Vassao DG, Lewis NG (2008) Dissection of lignin macromolecular configuration and assembly: comparison to related biochemical processes in allyl/propenyl phenol and lignan biosynthesis. Nat Prod Rep 25:1015-1090

D'Auria JC (2006) Acyltransferases in plants: a good time to be BAHD. Curr Opin Plant Biol 9:331-340

Dixon RA, Reddy MS, Gallego - Giraldo L (2014) Monolignol Biosynthesis and its Genetic Manipulation: The Good, the Bad, and the Ugly. Recent Advances in Polyphenol Research, Volume 4 1-38

Ehlting J, Büttner D, Wang Q, Douglas CJ, Somssich IE, Kombrink E (1999) Three 4 - coumarate: coenzyme A ligases in Arabidopsis thaliana represent two evolutionarily divergent classes in angiosperms. The Plant Journal 19:9-20

Eudes A, Pollet B, Sibout R, Do CT, Seguin A, Lapierre C, Jouanin L (2006) Evidence for a role of AtCAD 1 in lignification of elongating stems of Arabidopsis thaliana. Planta 225:23-39

Franke R, Hemm MR, Denault JW, Ruegger MO, Humphreys JM, Chapple C (2002) Changes in secondary metabolism and deposition of an unusual lignin in the ref8 mutant of Arabidopsis. The Plant Journal 30:47-59

Gao W, Li HY, Xiao S, Chye ML (2010) Acyl - CoA - binding protein 2 binds lysophospholipase 2 and lysoPC to promote tolerance to cadmium - induced oxidative stress in transgenic Arabidopsis. The Plant Journal 62:989-100 Grabherr MG, Haas BJ, Yassour M, Levin JZ, Thompson DA, Amit I, Adiconis X, Fan L, Raychowdhury R, Zeng QD, Chen ZH, Mauceli E, Hacohen N, Gnirke A, Rhind N, di Palma F, Birren BW, Nusbaum C, Lindblad-Toh K, Friedman N, Regev A (2011) Full-length transcriptome assembly from RNA-Seq data without a reference genome. Nat Biotechnol 29:644-U130

Gui J, Shen J, Li L (2011) Functional characterization of evolutionarily divergent 4-coumarate:coenzyme a ligases in rice. Plant Physiol 157:574-586

Ha CM, Escamilla-Trevino L, Yarce JC, Kim H, Ralph J, Chen F, Dixon RA (2016) An essential role of caffeoyl shikimate esterase in monolignol biosynthesis in Medicago truncatula. Plant J 86:363-375

Haider S, Azmat R (2012) Failure of Survival Strategies in Adaption of Heavy Metal Environment in Lens Culinaris and Phaseolus Mungo. Pak J Bot 44:1959-1964

Hamberger B, Ellis M, Friedmann M, Clarice D, Barbazuk B, Douglas CJ (2007) Genome-wide analyses of phenylpropanoid-related genes in Populus trichocarpa, Arabidopsis thaliana, and Oryza sativa: the Populus lignin toolbox and conservation and diversification of angiosperm gene families. Canadian Journal of Botany 85:1182-1201 Hambidge M (2000) Human Zinc Deficiency. J Nutr 130:1344S

He N, Zhang C, Qi X, Zhao S, Tao Y, Yang G, Lee T, Wang X, Cai Q, Li D (2013) Draft genome sequence of the mulberry tree Morus notabilis. Nature Communications 4:2445-2445

Huang J, Gu M, Lai Z, Fan B, Shi K, Zhou Y-H, Yu J-Q, Chen Z (2010) Functional analysis of the Arabidopsis PAL gene family in plant growth, development, and response to environmental stress. Plant Physiol 153:1526-1538

Humphreys JM, Hemm MR, Chapple C (1999) New routes for lignin biosynthesis defined by biochemical characterization of recombinant ferulate 5-hydroxylase, a multifunctional cytochrome P450-dependent monooxygenase. Proc Natl Acad Sci U S A 96:10045-10050

Iseli C, Jongeneel CV, Bucher P (1999) ESTScan: a program for detecting, evaluating, and reconstructing potential coding regions in EST sequences. Proc Int Conf Intell Syst Mol Biol 138-148

Jiang Y, Huang R, Yan X, Jia C, Long T (2017) Mulberry for environmental protection.

PeerJ reviewing PDF | (2020:12:56482:4:0:NEW 5 Jul 2021) 
524

525

526

527

528

529

530

531

532

533

534

535

536

537

538

539

540

541

542

543

544

545

546

547

548

549

550

551

552

553

554

555

556

557

558

559

560

561

562

563

564

Jiao F, Luo R, Dai X, Liu H, Yu G, Han S, Lu X, Su C, Chen Q, Song Q, Meng C, Li F, Sun H, Zhang R, Hui T, Qian Y, Zhao A, Jiang $Y(2020)$ Chromosome-level reference genome and population genomic analysis provide insight into the evolution and improvement of domesticated mulberry (Morus alba L). Mol Plant

Khalid N, Fawad SA, Ahmed I (2011) Antimicrobial activity, phytochemical profile and trace minerals of black mulberry (Morus Nigra L.) fresh juice. Pak J Bot 43:91-96

Kim RJ, Kim HJ, Shim D, Suh MC (2016) Molecular and biochemical characterizations of the monoacylglycerol lipase gene family of Arabidopsis thaliana. Plant J 85:758-771

Kumar S, Stecher G, Tamura K (2016) MEGA7: Molecular Evolutionary Genetics Analysis Version 7.0 for Bigger Datasets. Molecular Biology \& Evolution 33:1870

Kumar Tewari R, Kumar P, Nand Sharma P (2008) Morphology and physiology of zinc-stressed mulberry plants. J Plant Nutr Soil Sci 171:286-294

Langdon WB (2015) Performance of genetic programming optimised Bowtie2 on genome comparison and analytic testing (GCAT) benchmarks. BioData Min 8:1

Lee Y, Chen F, Gallego-Giraldo L, Dixon RA, Voit EO (2011) Integrative analysis of transgenic alfalfa (Medicago sativa L.) suggests new metabolic control mechanisms for monolignol biosynthesis. PLoS Comp Biol 7:

Li L, Cheng XF, Leshkevich J, Umezawa T, Harding SA, Chiang VL (2001) The last step of syringyl monolignol biosynthesis in angiosperms is regulated by a novel gene encoding sinapyl alcohol dehydrogenase. Plant Cell 13:1567-1586Li T, Qi X, Zeng Q, Xiang Z, He N (2014) MorusDB: a resource for mulberry genomics and genome biology. Database 2014:

Li X, Weng J, Chapple C (2008) Improvement of biomass through lignin modification. Plant J 54:569-581

Li Y, Im Kim J, Pysh L, Chapple C (2015) Four Isoforms of Arabidopsis 4-Coumarate: CoA Ligase Have Overlapping yet Distinct Roles in Phenylpropanoid Metabolism. Plant Physiol 169:2409-2421

Łochyńska M (2015) Energy and nutritional properties of the white mulberry (Morus alba I.). Journal of Agricultural Science and Technology 5:709-716

Niggeweg R, Michael AJ, Martin C (2004) Engineering plants with increased levels of the antioxidant chlorogenic acid. Nat Biotechnol 22:746-754

Raes J, Rohde A, Christensen JH, De Peer YV, Boerjan W (2003) Genome-Wide Characterization of the Lignification Toolbox in Arabidopsis. Plant Physiol 133:1051-1071

Ragauskas AJ, Beckham GT, Biddy MJ, Chandra R, Chen F, Davis MF, Davison BH, Dixon RA, Gilna P, Keller M (2014) Lignin Valorization: Improving Lignin Processing in the Biorefinery. Science 344:1246843

Ramirez F, Dundar F, Diehl S, Gruning BA, Manke T (2014) deepTools: a flexible platform for exploring deepsequencing data. Nucleic Acids Res 42:W187-191

Rao G, Pan X, Xu F, Zhang Y, Cao S, Jiang X, Lu H (2015) Divergent and Overlapping Function of Five 4Coumarate/Coenzyme A Ligases from Populus tomentosa. Plant Molecular Biology Reporter 33:841-854

Saleme MLS, Cesarino I, Vargas L, Kim H, Vanholme R, Goeminne G, Van Acker R, Fonseca FCA, Pallidis A, Voorend W, Junior JN, Padmakshan D, Van Doorsselaere J, Ralph J, Boerjan W (2017) Silencing CAFFEOYL SHIKIMATE ESTERASE Affects Lignification and Improves Saccharification in Poplar. Plant Physiol 175:1040-1057

Schoch G, Goepfert S, Morant M, Hehn A, Meyer D, Ullmann P, and Werck-Reichhart D. 2001. CYP98A3 from Arabidopsis thaliana is a 3'-hydroxylase of phenolic esters, a missing link in the phenylpropanoid pathway. J Biol Chem 276:36566-36574

Shi R, Sun YH, Li Q, Heber S, Sederoff R, Chiang VL (2010) Towards a systems approach for lignin biosynthesis in

PeerJ reviewing PDF | (2020:12:56482:4:0:NEW 5 Jul 2021) 
565

566

567

568

569

570

571

572

573

574

575

576

577

578

579

580

581

582

583

584

585

586

587

588

589

590

591

592

593

594

595
Populus trichocarpa: transcript abundance and specificity of the monolignol biosynthetic genes. Plant Cell Physiol 51:144-163

Shukla P, Reddy RA, Ponnuvel KM, Rohela GK, Shabnam AA, Ghosh MK, Mishra RK (2019) Selection of suitable reference genes for quantitative real-time PCR gene expression analysis in Mulberry (Morus alba L.) under different abiotic stresses. Mol Biol Rep 46:1809-1817

Susheelamma BN, Shekar KR, Sarkar A, Rao MR, Datta RK (1996) Genotype and hormonal effects on callus formation and regeneration in mulberry. Euphytica 90:25-29

Tang Y, Liu Q, Chen F (2012) Preparation and characterization of activated carbon from waste ramulus mori. Chem Eng J 203:19-24

Umezawa T (2018) Lignin modification in planta for valorization. Phytochem Rev 17:1305-1327

van de Mortel JE, Almar Villanueva L, Schat H, Kwekkeboom J, Coughlan S, Moerland PD, Ver Loren van Themaat E, Koornneef M, Aarts MG (2006) Large expression differences in genes for iron and zinc homeostasis, stress response, and lignin biosynthesis distinguish roots of Arabidopsis thaliana and the related metal hyperaccumulator Thlaspi caerulescens. Plant Physiol 142:1127-1147

Vanholme R, Cesarino I, Rataj K, Xiao Y, Sundin L, Goeminne G, Kim H, Cross J, Morreel K, Araujo P (2013) Caffeoyl shikimate esterase (CSE) is an enzyme in the lignin biosynthetic pathway in Arabidopsis. Science 341:1103-1106 Wang CH, Yu J, Cai YX, Zhu PP, Liu CY, Zhao AC, Lu RH, Li MJ, Xu FX, Yu MD (2016) Characterization and Functional Analysis of 4-Coumarate:CoA Ligase Genes in Mul-berry. PLoS One 11:e0155814

Weng J-K, Li X, Stout J, Chapple C (2008) Independent origins of syringyl lignin in vascular plants. Proceedings of the National Academy of Sciences 105:7887-7892

Weng JK, Chapple C (2010) The origin and evolution of lignin biosynthesis. New Phytol 187:273-285

Yan X, Liu J, Kim H, Liu B, Huang X, Yang Z, Lin YJ, Chen H, Yang C, Wang JP, Muddiman DC, Ralph J, Sederoff RR, Li Q, Chiang VL (2019) CAD1 and CCR2 protein complex formation in monolignol biosynthesis in Populus trichocarpa. New Phytol 222:244-260

Whetten R, Sederoff R (1995) Lignin Biosynthesis. Plant Cell 7:1001-1013Yuan Q, Zhao L (2017) The Mulberry (Morus alba L.) Fruit-A Review of Characteristic Components and Health Benefits. J Agric Food Chem 65:10383-10394

Zabed H, Sahu JN, Boyce AN, Faruq G (2016) Fuel ethanol production from lignocellulosic biomass: An overview on feedstocks and technological approaches. Renewable \& Sustainable Energy Reviews 66:751-774 Zhao Q (2016) Lignification: flexibility, biosynthesis and regulation. Trends Plant Sci 21:713-721 Zhong R, lii WH, Negrel J, Ye ZH (1998) Dual methylation pathways in lignin biosynthesis. Plant Cell 10:2033-2046 
596

597

598

599

600

601

602

603

604

605

606

607

608

609

610

611

612

613

614

615

616

617

618

619

620

\section{Figure Legends}

Figure 1. Phylogenetic analysis and expression profile of PAL and 4CL gene family in mulberry.

(A). Phylogenetic analysis of PALs; (B). Expression profiles of PAL gene family in different tissues or organs in Morus notabilis and Fengchi; (C). Phylogenetic analysis of 4CLs; (D). Expression profiles of 4CL gene family in different tissues or organs in Morus notabilis and Fengchi. Red full circles indicating PALs or 4CLs from dicots, blue full circles indicating PALs or 4CLs from monocots, green full circles indicating PALs or 4CLs from gymnosperms and yellow full circles indicating PALs from ferns or moss. Putative protein sequences were used for phylogenetic analysis and the sequences information is available in Table S2. Mn indicating Morus notabilis and Fc indicating Fengchi. L0, leaf without Zinc treatment; S0, stem without zinc treatment; R0, root without zinc treatment. Bona fide clades were marked using different color shading

Figure 2. Phylogenetic analysis and expression profile of $\mathrm{C3}^{\prime} \mathrm{H}$ and $\mathrm{C4H}$ gene families in mulberry.

(A). Phylogenetic analysis of C4Hs; (B). Phylogenetic analysis of C3'Hs. c. Expression profiles of $\mathrm{C}^{\prime}{ }^{\mathrm{H}}$ and $\mathrm{C} 4 \mathrm{H}$ gene family in different tissues or organs in Morus notabilis and Fengchi. Red full circles indicating proteins from dicots, blue full circles indicating proteins from monocots, green full circles indicating proteins from gymnosperms and yellow full circles indicating proteins from ferns or moss. Bona fide clades were marked using different color shadings. Putative protein sequences were used for phylogenetic analysis and the sequences information is available in Table S2. Mn indicating Morus notabilis and Fc indicating Fengchi. L0, leaf without zinc treatment; S0, stem without zinc treatment; R0, root without zinc treatment. 
622 Figure 3. Phylogenetic analysis and expression profile of HCT and CSE gene families in 623 mulberry.

624 (A). Phylogenetic analysis of HCTs; (B). Phylogenetic analysis of CSEs; (C). Expression profiles 625 of HCT and CSE gene family in different tissues or organs in Morus notabilis and Fengchi. Red 626 full circles indicating proteins from dicots, red empty circles indicating HQTs, blue full circles 627 indicating proteins from monocots, green full circles indicating proteins from gymnosperms and 628 yellow full circles indicating proteins from ferns or moss. Bona fide clades were marked using 629 different color shadings. Putative protein sequences were used for phylogenetic analysis and the 630 sequences information is available in Table S2. Mn indicating Morus notabilis and Fc indicating 631 Fengchi. L0, leaf without zinc treatment; S0, stem without zinc treatment; R0, root without zinc 632 treatment.

633 profiles of CCOAOMT and COMT gene family in different tissues or organs in Morus notabilis and Fengchi. Red full circles indicating proteins from dicots, blue full circles indicating proteins from monocots, green full circles indicating proteins from gymnosperms and yellow full circles indicating proteins from ferns or moss. Bona fide clades were marked using different color shadings. Putative protein sequences were used for phylogenetic analysis and the sequences 642 information is available in Table S2. Mn indicating Morus notabilis and Fc indicating Fengchi. L0, leaf without zinc treatment; S0, stem without zinc treatment; R0, root without zinc treatment. 
645

646

647

648

649

650

651

652

653

654

655

656

657

658

659

660

661

662

663

664

665

666

Figure 5. Phylogenetic analysis and expression profile of $\mathrm{F} 5 \mathrm{H}$ gene family in mulberry.

(A). Phylogenetic analysis of F5Hs; (B). Expression profiles of F5H gene family in different tissues or organs in Morus notabilis and Fengchi. Red full circles indicating F5Hs from dicots, blue full circles indicating F5Hs from monocots, green full circles indicating F5Hs from gymnosperms and yellow full circles indicating F5Hs from ferns or moss. Bona fide clades were marked using different color shadings. Putative protein sequences were used for phylogenetic analysis and the sequences information is available in Table S2. Mn indicating Morus notabilis and Fc indicating Fengchi. L0, leaf without zinc treatment; S0, stem without zinc treatment; R0, root without zinc treatment.

\section{Figure 6. Phylogenetic analysis and expression profile of CCR and CAD gene families in} mulberry.

(A). Phylogenetic analysis of CCRs; (B). Phylogenetic analysis of CADs; (C). Expression profiles of CCR and CAD gene family in different tissues or organs in Morus notabilis and Fengchi. Red full circles indicating proteins from dicots, blue full circles indicating proteins from monocots, green full circles indicating proteins from gymnosperms and yellow full circles indicating proteins from ferns or moss. Bona fide clades were marked using different color shadings. Putative protein sequences were used for phylogenetic analysis and the sequences information is available in Table S2. Mn indicating Morus notabilis and Fc indicating Fengchi. L0, leaf without zinc treatment; S0, stem without zinc treatment; R0, root without zinc treatment. 
668 Figure 7. Expression change of bona fide clade genes in response to excess zinc stress in 669 mulberry.

670 (A). Fold change of expression levels of 23 bona fide genes in Fengchi after excess zinc treatment;

671 (B). Overall change of monolignol pathway in different organs after excess zinc treatment in 672 Fengchi; (C). Clustering of 23 bona fide clade genes expression pattern in response to Zinc stress.

673 Two biological replicates with three technical replicates respectively were performed for RT674 qPCR. P-value was calculate using SPSS 19.0. “*” indicates $0.01<\mathrm{p}<0.05$; “**” indicates $6750.001<\mathrm{p}<0.01$ and “****" indicates $\mathrm{p}<0.001$.

676 Figure S1 Pro concentration and SOD concentration in mulberry leaves, roots and stems at

67715 days after treatment

678 Figure S2 The melt curve of each gene for RT-qPCR experiments

679 Figure S3 RT-qPCR results for 23 bona fide clade genes in Fengchi

680 Figure S4 RT-qPCR for FcF5H3(Sm) and FcF5H4(Sm) in Fengchi

681 Figure S5 Alignment and motif analysis of CCRs. The motifs were marked in red box.

682 Figure S6 Expression profile of all candidate genes in mulberry.

683 (A). Hierarchical clustering of expression profiles of 56 candidate genes based on transcriptome 684 data in mulberry; Blue star indicated the cluster I and red star indicate the cluster II. Red full circles 685 indicated the bona fide clade genes (B). Expression profiles of 23 bona fide clade genes in Fengchi. 686 


\section{Figure 1}

Figure 1. Phylogenetic analysis and expression profile of PAL and $4 C L$ gene family in mulberry.

(A). Phylogenetic analysis of PALs; (B). Expression profiles of PAL gene family in different tissues or organs in Morus notabilis and Fengchi; (C). Phylogenetic analysis of 4CLs; (D). Expression profiles of $4 C L$ gene family in different tissues or organs in Morus notabilis and Fengchi. Red full circles indicating PALs or 4CLs from dicots, blue full circles indicating PALs or 4CLs from monocots, green full circles indicating PALs or 4CLs from gymnosperms and yellow full circles indicating PALs from ferns or moss. Putative protein sequences were used for phylogenetic analysis and the sequences information is available in Table S2 . Mn indicating Morus notabilis and Fc indicating Fengchi. L0, leaf without Zinc treatment; S0, stem without zinc treatment; R0, root without zinc treatment. Bona fide clades were marked using different color shading 


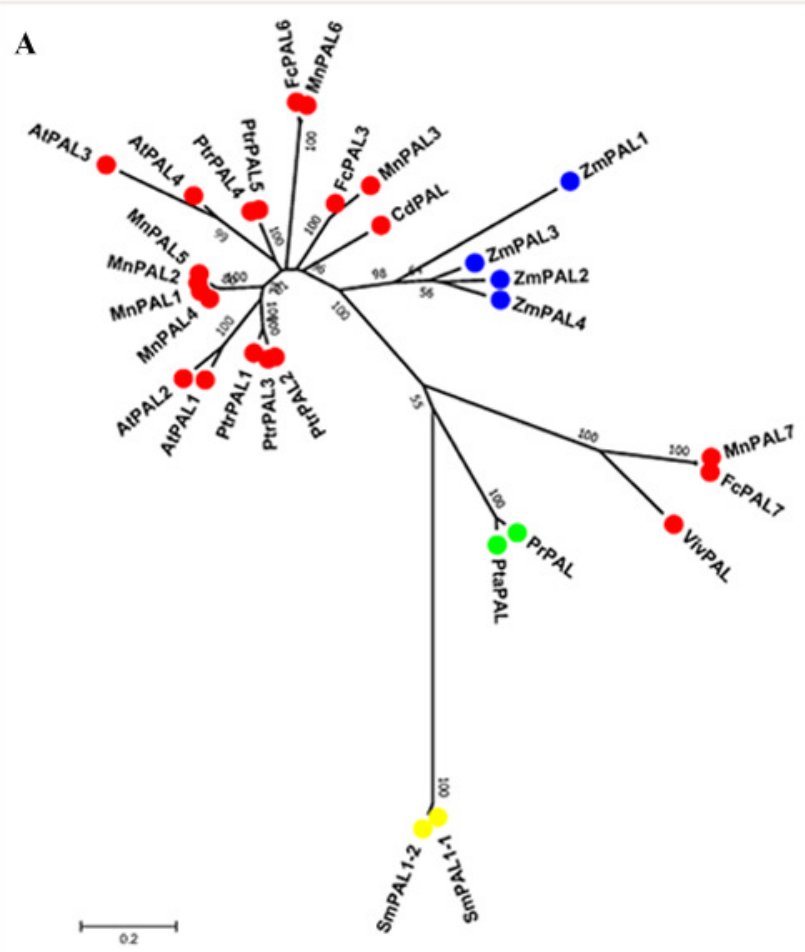

B

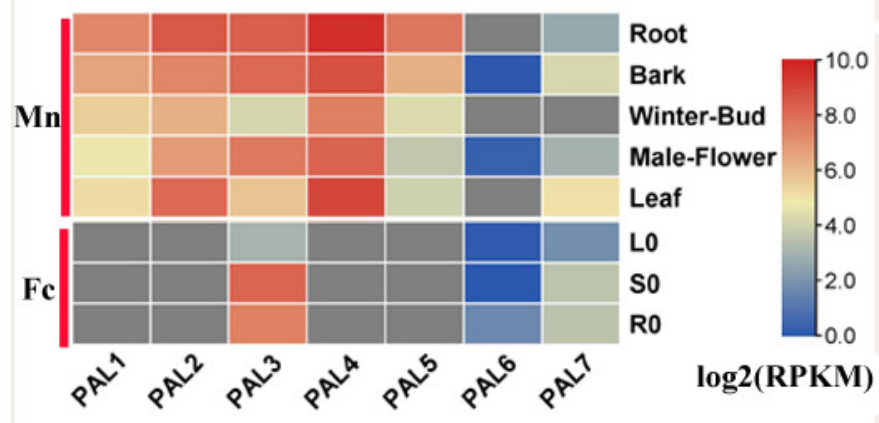

C

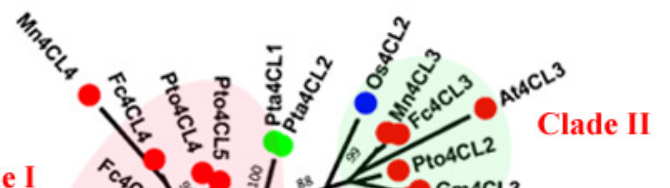

Clade I

Clade
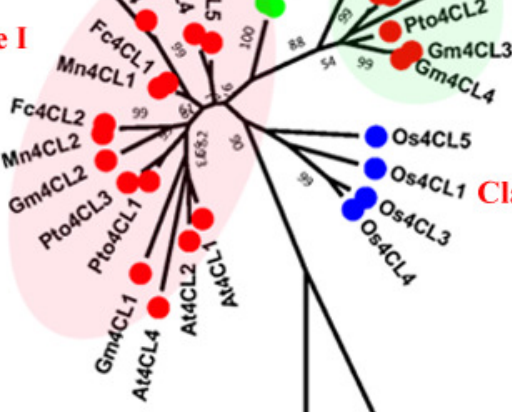

OSACL5

西
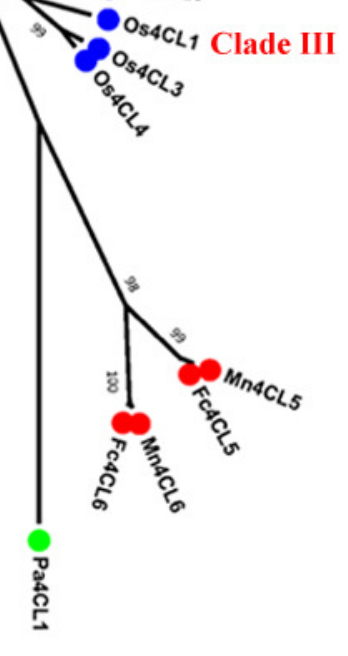

D

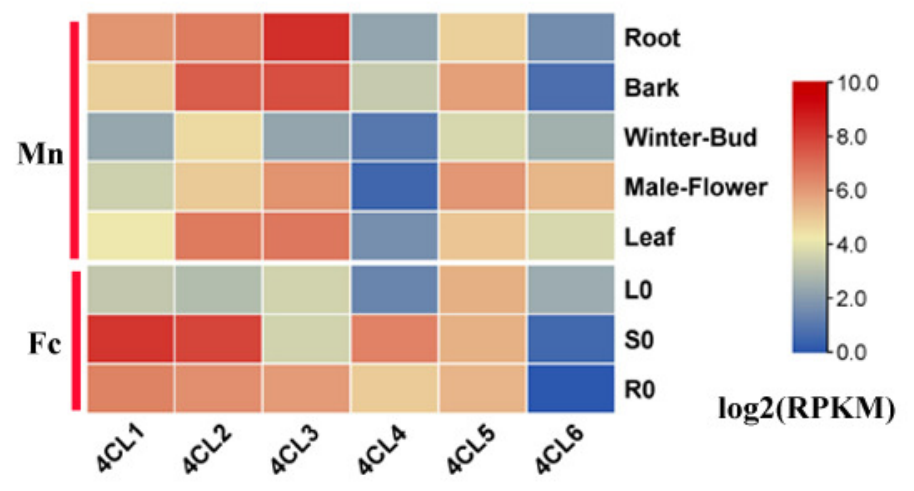




\section{Figure 2}

Figure 2. Phylogenetic analysis and expression profile of $\mathrm{C}^{\prime} \mathrm{H}$ and $\mathrm{C} 4 \mathrm{H}$ gene families in mulberry.

(A). Phylogenetic analysis of C4Hs; (B). Phylogenetic analysis of C3'Hs. (C). Expression profiles of $\mathrm{C}^{\prime} \mathrm{H}$ and $\mathrm{C} 4 \mathrm{H}$ gene family in different tissues or organs in Morus notabilis and Fengchi. Red full circles indicating proteins from dicots, blue full circles indicating proteins from monocots, green full circles indicating proteins from gymnosperms and yellow full circles indicating proteins from ferns or moss. Bona fide clades were marked using different color shadings. Putative protein sequences were used for phylogenetic analysis and the sequences information is available in Table S2. Mn indicating Morus notabilis and Fc indicating Fengchi. L0, leaf without zinc treatment; S0, stem without zinc treatment; R0, root without zinc treatment. 


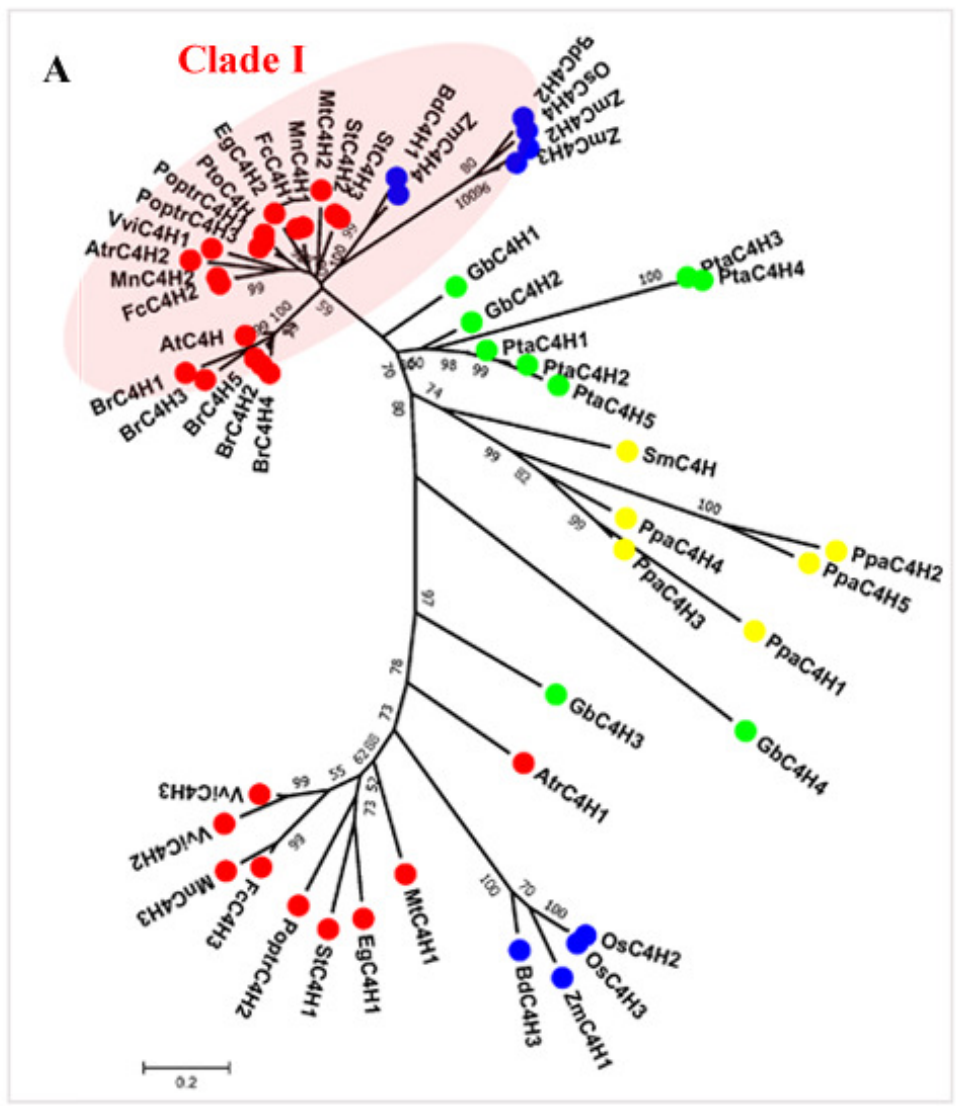

B

C
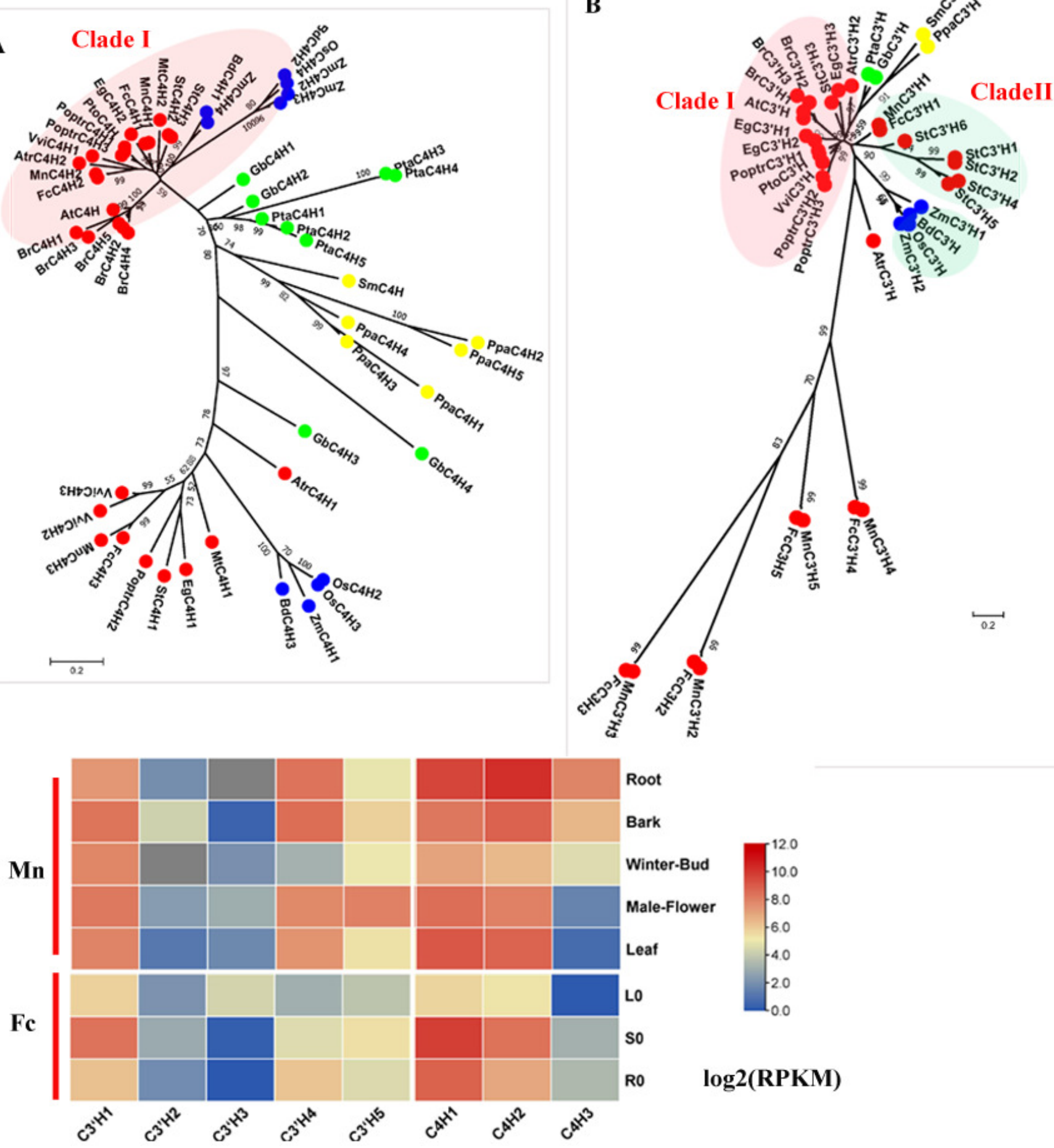


\section{Figure 3}

Figure 3. Phylogenetic analysis and expression profile of HCT and CSE gene families in mulberry.

(A). Phylogenetic analysis of HCTs; (B). Phylogenetic analysis of CSEs; (C). Expression profiles of HCT and CSE gene family in different tissues or organs in Morus notabilis and Fengchi . Red full circles indicating proteins from dicots, red empty circles indicating HQTs, blue full circles indicating proteins from monocots, green full circles indicating proteins from gymnosperms and yellow full circles indicating proteins from ferns or moss. Bona fide clades were marked using different color shadings. Putative protein sequences were used for phylogenetic analysis and the sequences information is available in Table S2. Mn indicating Morus notabilis and Fc indicating Fengchi. LO, leaf without zinc treatment; S0, stem without zinc treatment; R0, root without zinc treatment. 

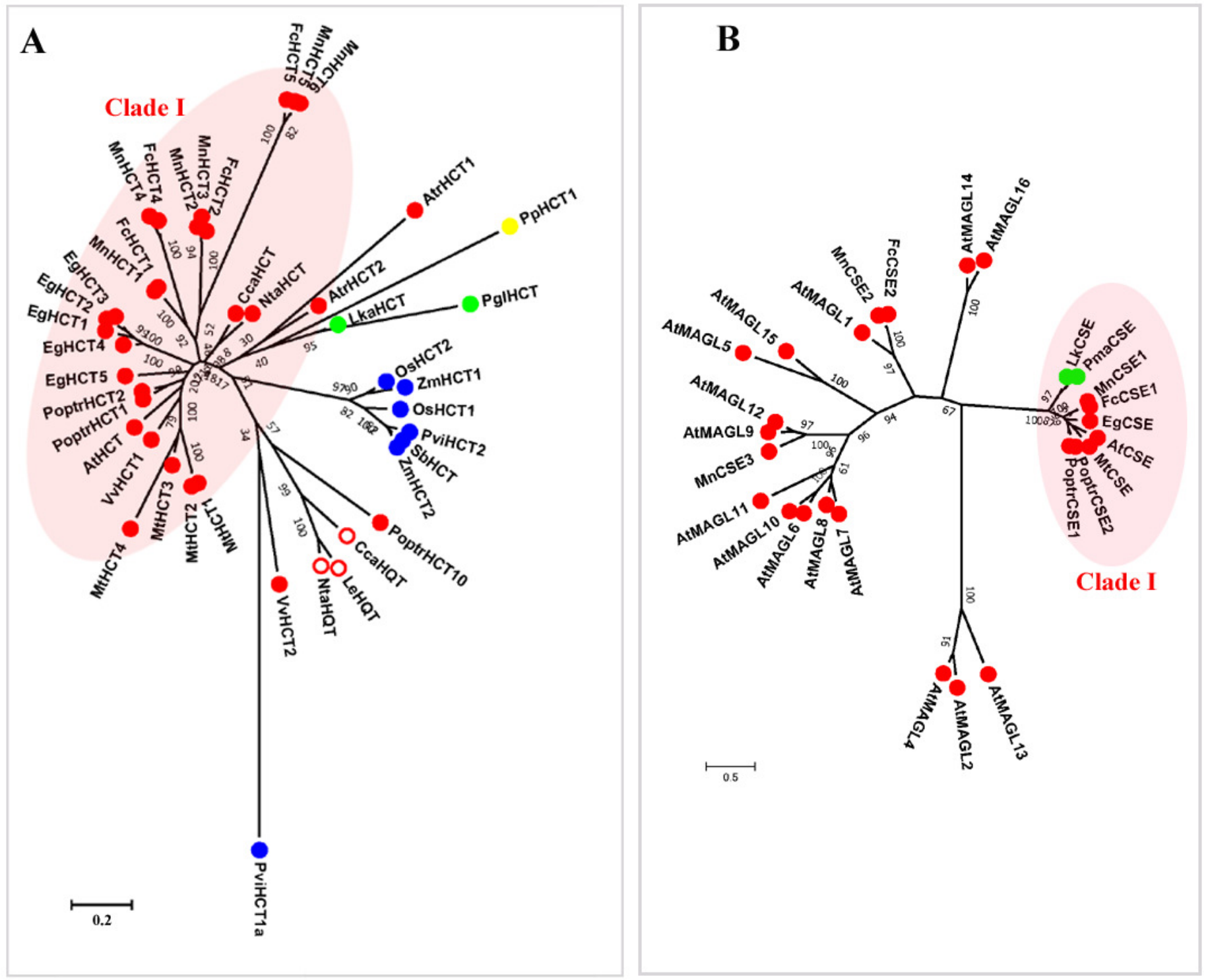

C

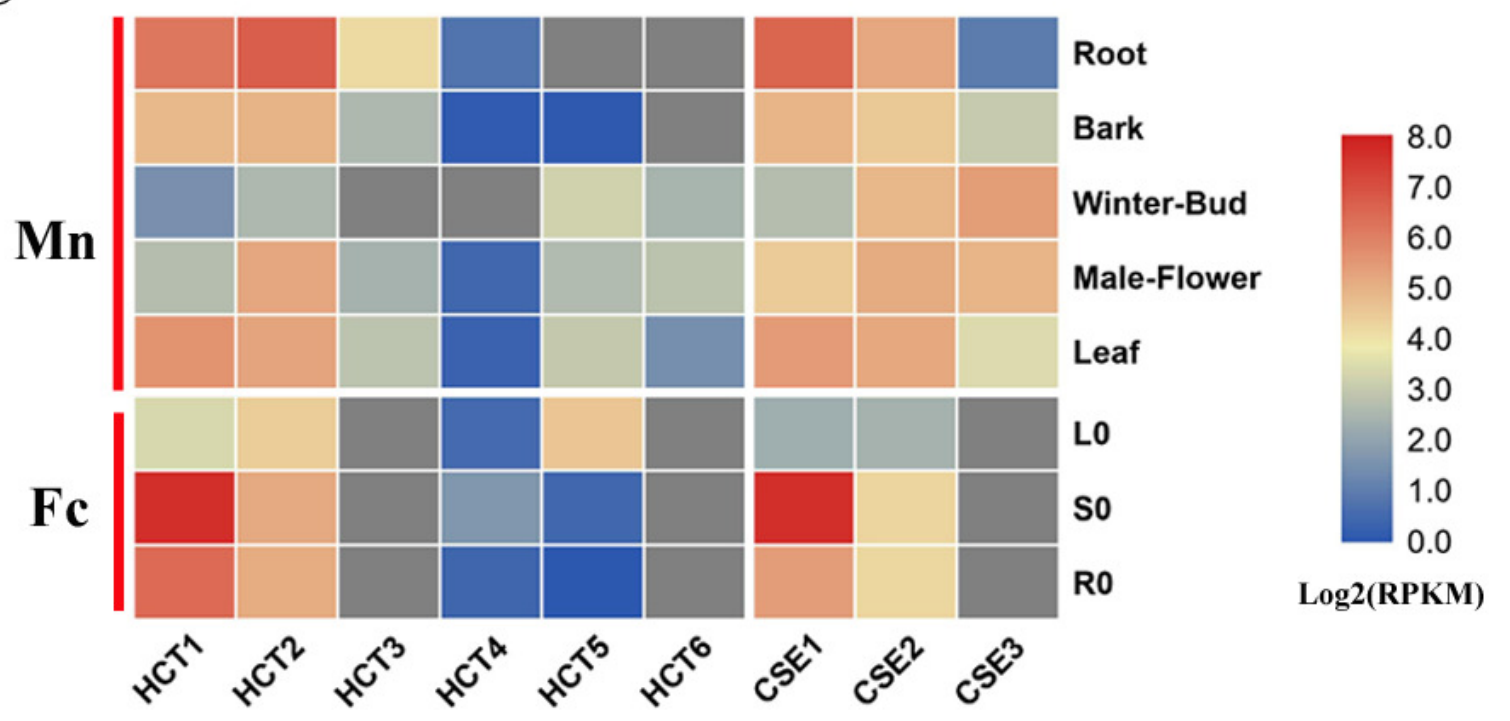




\section{Figure 4}

Figure 4. Phylogenetic analysis and expression profile of CCOAOMT and COMT gene families in mulberry.

(A). Phylogenetic analysis of CCOAOMTs; (B). Phylogenetic analysis of COMTs; (C). Expression profiles of CCOAOMT and COMT gene family in different tissues or organs in Morus notabilis and Fengchi. Red full circles indicating proteins from dicots, blue full circles indicating proteins from monocots, green full circles indicating proteins from gymnosperms and yellow full circles indicating proteins from ferns or moss. Bona fide clades were marked using different color shadings. Putative protein sequences were used for phylogenetic analysis and the sequences information is available in Table S2. Mn indicating Morus notabilis and Fc indicating Fengchi. L0, leaf without zinc treatment; S0, stem without zinc treatment; R0, root without zinc treatment. 


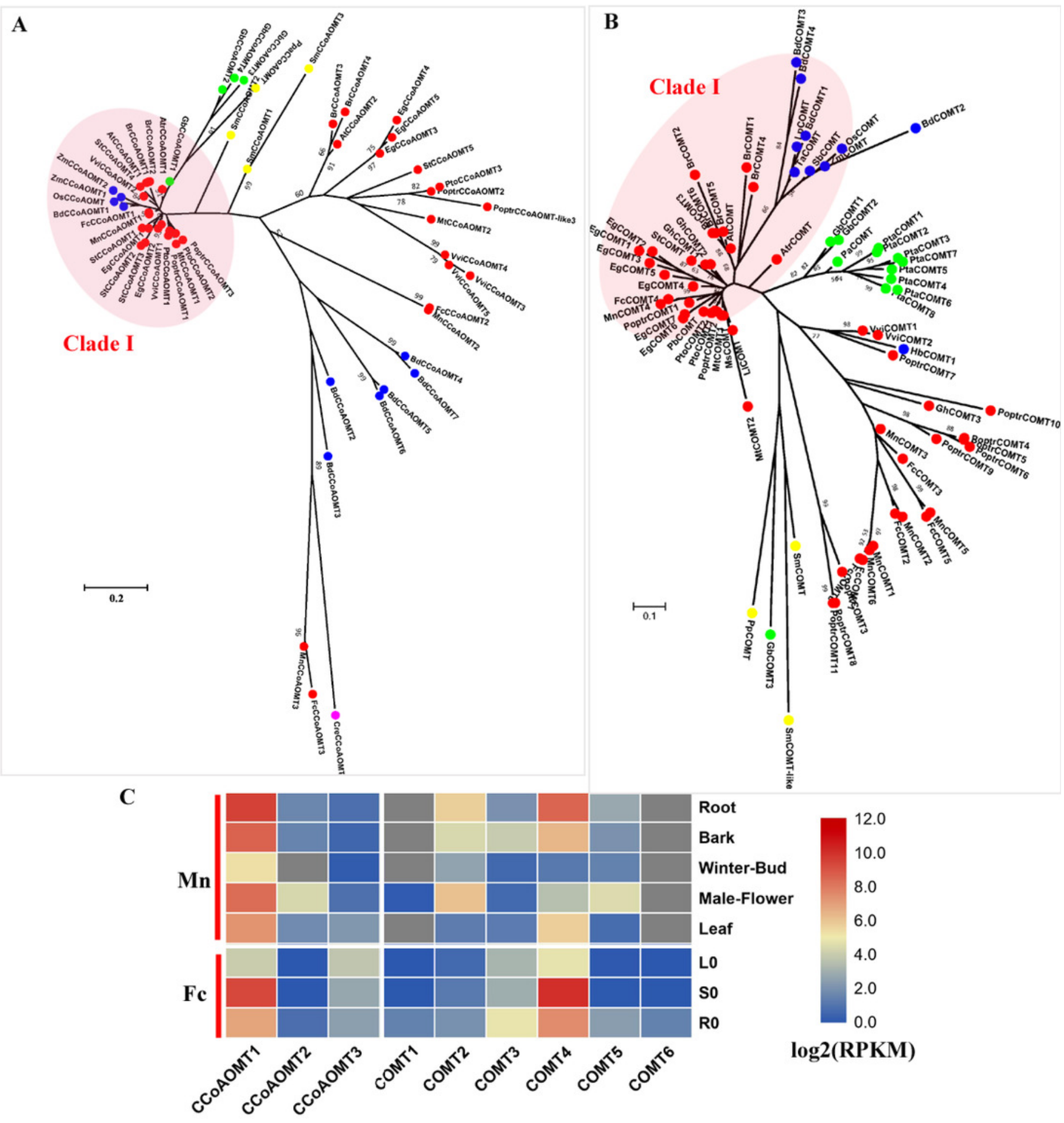




\section{Figure 5}

Figure 5. Phylogenetic analysis and expression profile of $\mathrm{F} 5 \mathrm{H}$ gene family in mulberry.

(A). Phylogenetic analysis of $\mathrm{F} 5 \mathrm{Hs}$; (B). Expression profiles of $\mathrm{F} 5 \mathrm{H}$ gene family in different tissues or organs in Morus notabilis and Fengchi. Red full circles indicating F5Hs from dicots, blue full circles indicating F5Hs from monocots, green full circles indicating F5Hs from gymnosperms and yellow full circles indicating F5Hs from ferns or moss. Bona fide clades were marked using different color shadings. Putative protein sequences were used for phylogenetic analysis and the sequences information is available in Table S2. Mn indicating Morus notabilis and Fc indicating Fengchi. LO, leaf without zinc treatment; S0, stem without zinc treatment; R0, root without zinc treatment. 


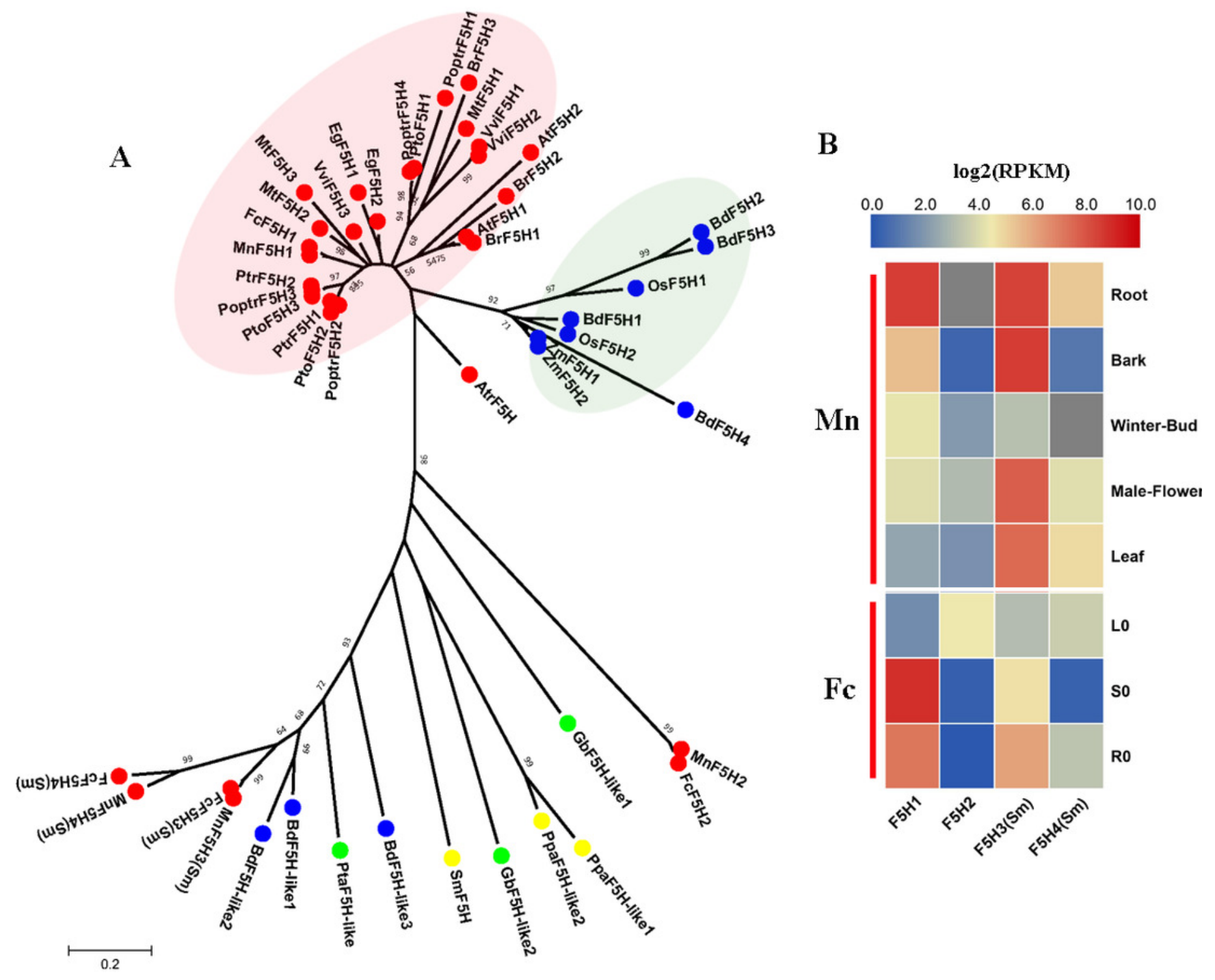




\section{Figure 6}

Figure 6. Phylogenetic analysis and expression profile of CCR and CAD gene families in mulberry.

(A). Phylogenetic analysis of CCRs; (B). Phylogenetic analysis of CADs; (C). Expression profiles of $C C R$ and $C A D$ gene family in different tissues or organs in Morus notabilis and Fengchi. Red full circles indicating proteins from dicots, blue full circles indicating proteins from monocots, green full circles indicating proteins from gymnosperms and yellow full circles indicating proteins from ferns or moss. Bona fide clades were marked using different color shadings. Putative protein sequences were used for phylogenetic analysis and the sequences information is available in Table S2. Mn indicating Morus notabilis and Fc indicating Fengchi. L0, leaf without zinc treatment; S0, stem without zinc treatment; R0, root without zinc treatment. 


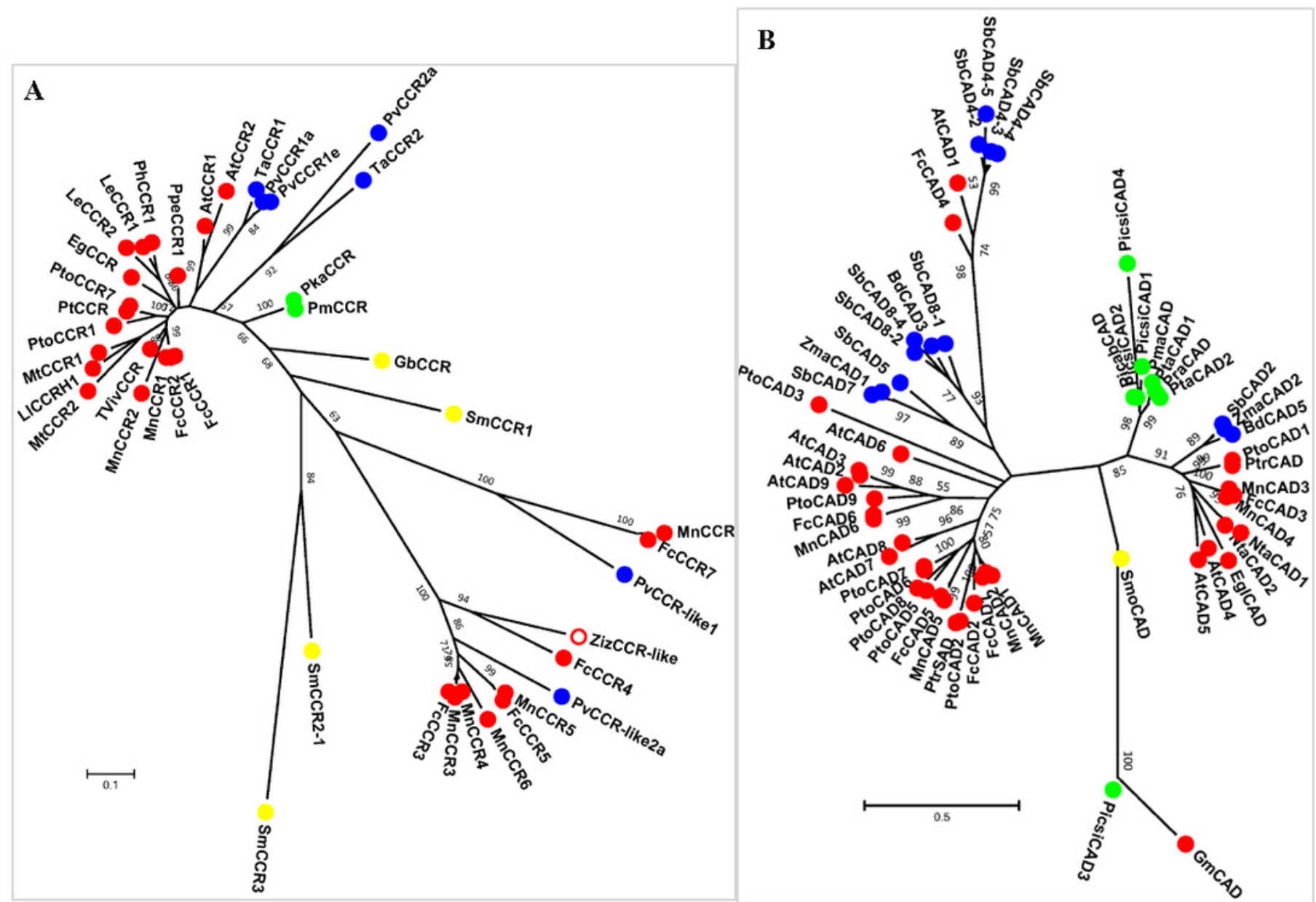

C

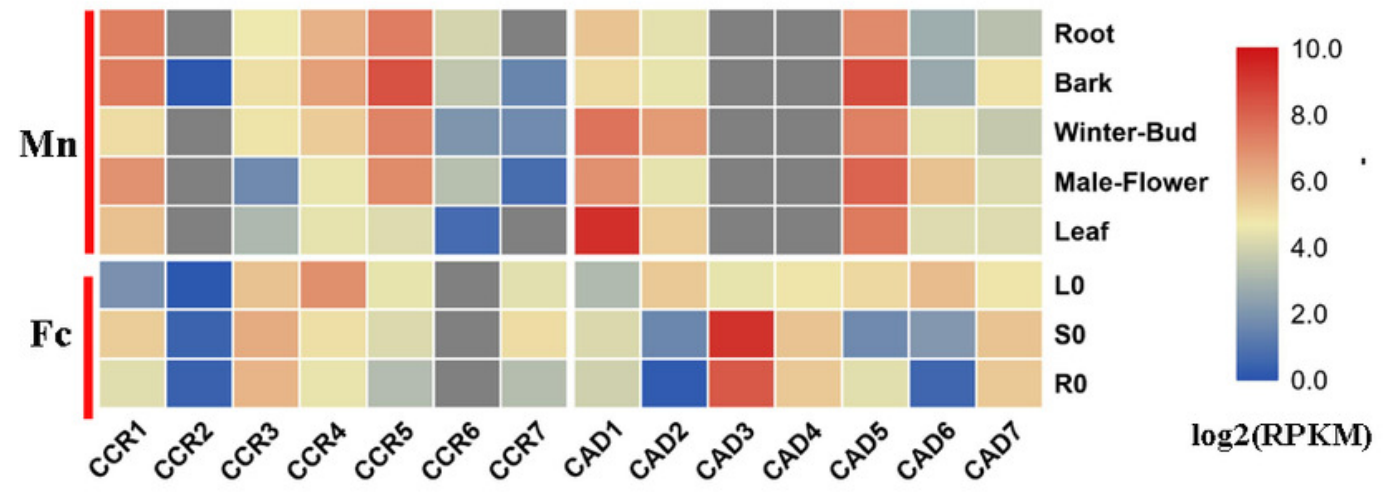




\section{Figure 7}

Figure 7. Expression change of bona fide clade genes in response to excess zinc stress in mulberry.

(A). Fold change of expression levels of 23 bona fide genes in Fengchi after excess zinc treatment; (B). Overall change of monolignol pathway in different organs after excess zinc treatment in Fengchi ; (C). Clustering of 23 bona fide clade genes expression pattern in response to Zinc stress. Two biological replicates with three technical replicates respectively were performed for qRT-PCR. P-value was calculate using SPSS 19.0. “*” indicates $0.01<p<0.05 ;$; $* *$ " indicates $0.001<p<0.01$ and "***" indicates $p<0.001$. 


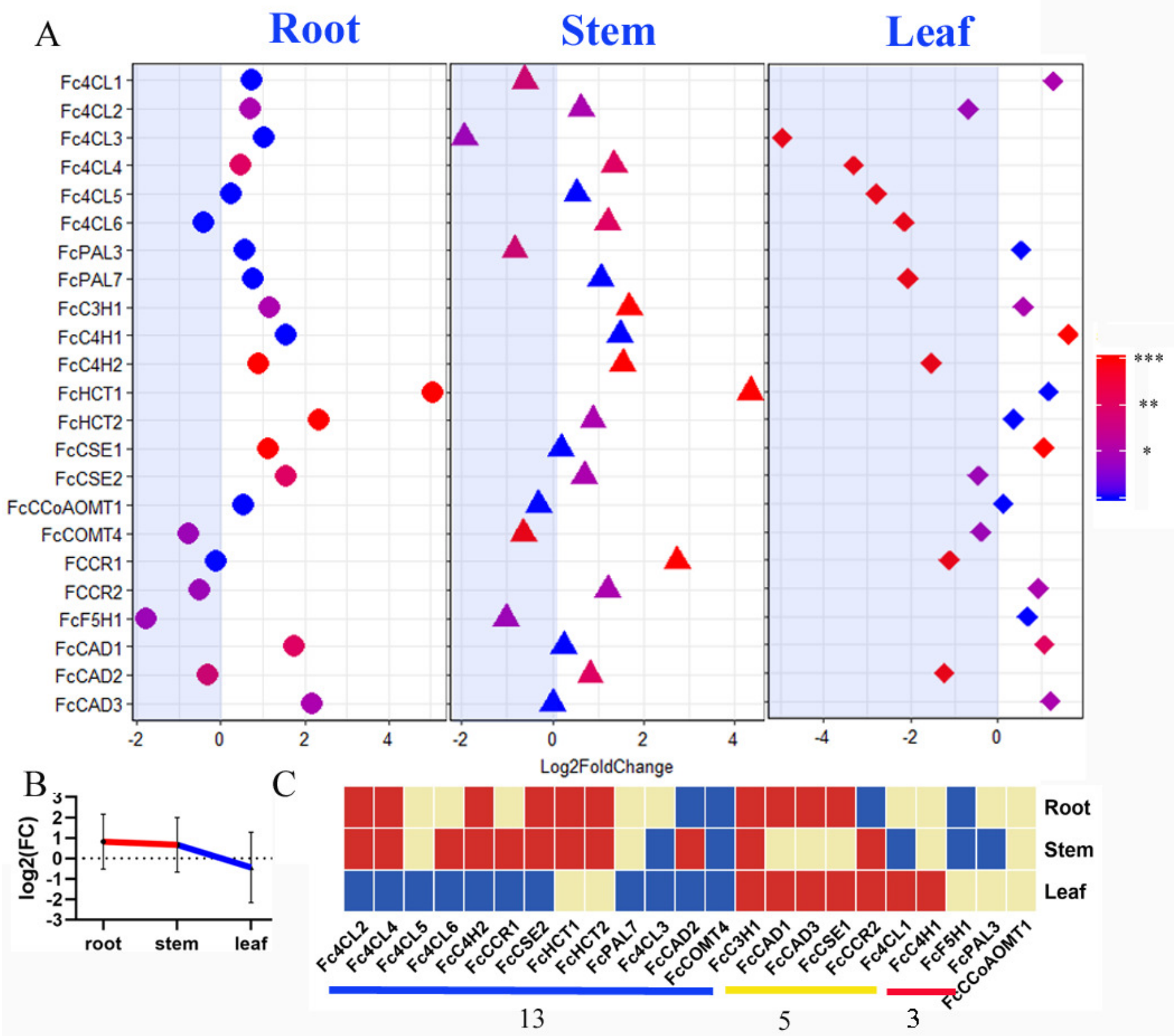

